\title{
Graph Theory assisted Bit-to-Index-Combination Gray Coding for Generalized Index Modulation
}

\author{
Lixia Xiao, Member, IEEE, Da Chen, Member, IEEE, Ibrahim Hemadeh, Member, IEEE, Pei Xiao Senior \\ member, IEEE, and Tao Jiang, Fellow, IEEE
}

\begin{abstract}
Generalized index modulation (GIM) which implicitly conveys information by the activated indices is a promising technique for next-generation wireless networks. Due to the prohibitive challenge of bit-to-index combination (IC) mapping optimization, conventional GIM system obtains the bit-to-IC mapping table randomly, which may suffer from some performance loss. To circumvent this issue, we propose a low-complexity graph theory assisted bit-to-IC gray coding for GIM systems by minimizing the average hamming distance (HD) between any two ICs having one different value. Specifically, we decompose and transform the optimization problem into two subproblems using the graph theory, i.e., 1) Select an IC set whose corresponding graph has the minimum degree; 2) Design a bit-to-IC mapping principle to minimize the weight of the selected graph. Lowcomplexity algorithms are developed to solve the subproblems with a significant reduced complexity. Both simulation and theoretical results are shown that the GIM systems with our proposed mapping table are capable of providing significant performance gains over the conventional counterparts without the need for any additional feedback-link and without extra computational complexity. It is also shown that the proposed bit-to-IC mapping table is straightforward for any GIM systems over generalized fading channels.
\end{abstract}

Index Terms-Index modulation, graph theory, bit-to-indexcombination, gray coding.

\section{INTRODUCTION}

A $S$ a spectrum and energy efficient yet simple digital modulation scheme, generalized index modulation (GIM) employs the activate indices as an additional means to transmit information [1]-[2]. Since GIM exploits a completely new domain to increase the spectral efficiency without increasing the hardware complexity, it has been widely employed in domains such as transmit antennas (TAs), time slots, dispersion matrices, subcarriers, radio frequency (RF) mirrors, transmit light emitting diodes, relays, modulation types, precoder matrices, spreading codes, signal powers, loads and so on [1]-[9].

The concept of GIM was first employed in the TA domain, namely spatial modulation (SM), where only a single TA is

This work was supported in part by the National Key R\&D Program of China with Grant number 2019YFB180003400, National Science Foundation of China with Grant number 61831013, 61631015, and Innovation Fund of WNLO.

L. Xiao is with the Wuhan National Laboratory for Optoelectronics, Huazhong University of Science and Technology, Wuhan 430074, China (email: lixiaxiao@hust.edu.cn);

D. Chen, and T. Jiang are with the Wuhan National Laboratory for Optoelectronics and School of Electronic Information and Communications, Huazhong University of Science and Technology, Wuhan 430074, China (email: chenda@ hust.edu.cn; Tao.Jiang @ieee.org).

I. Hemadeh and P. Xiao are with 5G Innovation Center of University of Surrey, GU27XH UK (e-mail: ibrahimhemadeh@gmail.com; p.xiao@surrey.ac.uk). activated to transmit one amplitude phase modulation (APM) symbol [10]-[14]. To increase the throughput, generalized SM (GSM) was proposed in [15]-[16], where $N_{u}$ out of $N_{t}$ TAs are activated to transmit $N_{u}$ APM symbols, and is capable of achieving flexible transmission rate by configuring antenna setups. Researches have demonstrated that GSM is one of the promising candidates in millimeter wave channels and massive multiple-input multiple-output (MIMO) communication scenarios [17]-[24]. In order to exploit the diversity of SM, space time block coded (STBC) based SM was proposed in [25], where $N_{u}$ out of $N_{t}$ TAs are activated to transmit $N_{u} \times N_{u}$ STBC symbols. In the dispersion matrices domain, generalized space time shift keying (GSTSK) was proposed in [26]-[29], where $P$ out of $Q$ preassigned space-time dispersion matrices are activated with the aid of $P$ APM symbols. To further increase the transmission rate of GSTSK, multi-set GSTSK (MS-GSTSK) was proposed in [1], where $N_{u}$ out of $N_{t}$ TAs are activated to transmit a single GSTSK codeword. In summary, the GIM based MIMO system is capable of striking a flexible tradeoff between the spatial multiplexing gain and the spatial diversity gain.

To further exploit the benefits of GIM, orthogonal frequency division multiplexing (OFDM) with index modulation (OFDM-IM) was proposed in [30], which employs the activated subcarrier indices to transmit information. Since only part of subcarriers are utilized to transmit APM symbols, OFDM-IM exhibits lower peak average power ratio (PAPR) and inter carrier interference than the conventional OFDM [31]. Therefore, OFDM-IM achieves higher spectral efficiency and is more robust against the frequency selective channel. Due to the above advantages, numerous enhanced variants of OFDM-IM were proposed in [32]-[40].

However, the existing state-of-the-art of GIM is mainly focused on the performance analysis of the transceiver design. Since $N_{u}$ out of $N_{t}$ elements are activated to transmit information in a GIM system, there are a total of $N_{\text {all }}=C_{N_{t}}^{N_{u}}$ index combinations (ICs) available, and $N=2^{\left\lfloor\log _{2}\left(N_{\text {all }}\right)\right\rfloor}$ out of $N_{\text {all }}$ ICs are selected for bit-to-IC mapping, which can be seen in Fig. 1 (a). As shown in Fig. 1 (b), to find the optimal IC set and the best mapping principle with the optimal average bit error probability (ABEP), $C_{N_{\text {all }}}^{N} N$ ! ABEPs have to be calculated, which makes the bit-to-IC mapping prohibitively complicated and challenging.

Recently, some novel bit-to-symbol mapping principles were developed for the SM system $\left(N_{u}=1\right)$ in [41]-[43]. However, they are not suitable for GIM systems $\left(N_{u}>1\right)$. As a result, in existing GIM researches, both the IC selection 
and the mapping principle are randomly selected, resulting in performance loss. Although there existed a novel bit-tosymbol mapping for GSM based Euclidean distance in [44], it relies on the feedback of channel state information (CSI), which imposes substantial link budget and extra complexity. To the best of authors' knowledge, there is no general gray coding guideline for GIM systems without CSI feedback.

Against the above backgrounds, we propose a lowcomplexity bit-to-IC gray coding for GIM with the aid of graph theory [45] in this paper, where the bit-to-IC optimization problem is formulated by utilizing the idea of gray coding to minimize the average Hamming distance (HD) of any two ICs with only one different element. Specifically, we firstly transform each selected IC set with a specific mapping principle into a weighted graph and transform the optimization objective into obtaining one weighted graph with the minimum weight from all weighted graphs, where the weight is the HD of information bits between two adjacent vertexes. As shown in Fig. 1 (c), the optimization problem is decomposed into two subproblems, i.e., 1) Select an IC set whose corresponding graph has the minimum degree; 2) Design a bit-to-IC mapping principle to minimize the weight of the selected graph. The specific contributions of this paper are summarized as follows.

1) We transform the mapping issues into graph theory assisted optimizations and design a maximum degree first (MDF) based low-complexity algorithm with the complexity order of $O(N)$. We also demonstrate that it is comparable to the optimal algorithm using the mathematical techniques of induction.

2) We consider the original $N$ blocks of information bits as a completed weighted graph, and transform the bitto-IC mapping into finding the IC graph with the minimum weight. To solve the problem, we design a lowcomplexity minimum weight first (MWF) algorithm with the complexity order of $O\left(N^{2}\right)$.

3) The proposed bit-to-IC mapping principle is straightforward for any GIM systems over generalized fading channel. Moreover, both theoretical and simulation results show that the GIM system employing our proposed method achieves significant performance gains over the conventional counterparts without additional feedback link and without computational complexity.

The remainder of this paper is organized as follows. Section II gives a brief introduction to classic GIM systems and formulates the mapping problem. Section III presents the problem formulation with the aid of graph theory. Section IV introduces the optimization of the IC selection, while the optimization of the bit-to-IC mapping is presented in Section V. Simulation results are presented in Section VI. Finally, Section VII concludes this paper.

Notation: $\|\cdot\|_{2}$ denotes the two norm of a matrix. $|\cdot|$ represents the cardinality of a set. $(\cdot)^{*},(\cdot)^{T}$ and $(\cdot)^{H}$ stand for the conjugate, transpose and the Hermitian transpose of a vector/matrix, respectively. $x \gg y$ implies that $x$ is much larger than $y$ and $n$ ! denotes the factorial operator. $C_{m}^{n}$ is the binomial coefficient and $\lfloor\cdot\rfloor$ denotes the floor operator.

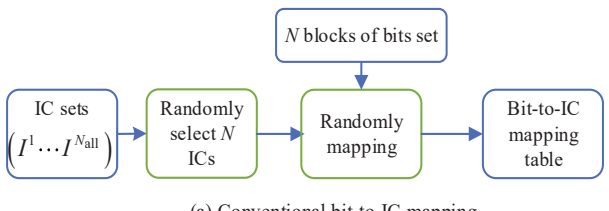

(a) Conventional bit-to-IC mapping

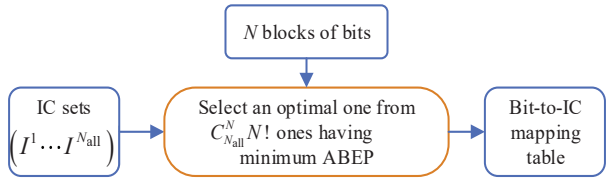

(b) Optimal bit-to-IC mapping

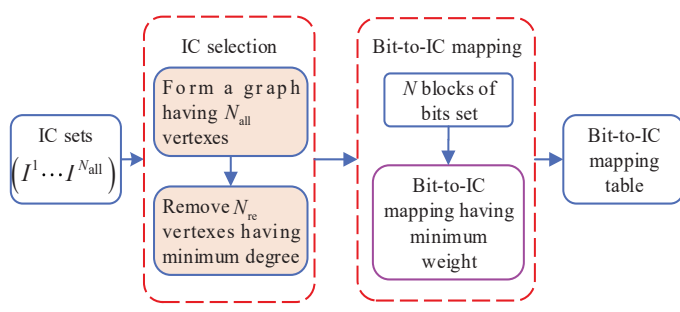

(c) Proposed bit-to-IC mapping

Fig. 1. Bit-to-IC mapping methods.

\section{PROBLEM FORMULATION}

\section{A. GIM system model}

In a GIM system, a block of information bits with length of $B$ is partitioned into two parts: 1) $B_{1}=\left\lfloor\log _{2}\left(C_{N_{t}}^{N_{u}}\right)\right\rfloor$ bits are used to select an IC $I^{i}=\left(i_{1}, \ldots, i_{N_{u}}\right) i=(1,2, \ldots, N)$; 2) $B_{2}=N_{u} \log _{2}(M)$ bits are used to modulate $N_{u} M$ APM symbols as $\mathbf{s}=\left(s_{i_{1}}, \ldots, s_{i_{N_{u}}}\right)$. Especially, we have $\mathbf{s}=(1, \ldots, 1)$ for the case of $M=1$. Hence, one GIM symbol can be expressed by [27]

$$
\mathbf{X}^{i}=\sum_{q=i_{1}}^{i_{N_{u}}} \mathbf{A}_{q} s_{q},
$$

where $\mathbf{A}_{q}$ is a dispersive matrix occupying $T$ time slots. Especially, $\mathbf{A}_{q}$ of GSM can be expressed by [27]

$$
\mathbf{A}_{q}=[\underbrace{0 \cdots 0}_{q-1} 1 \underbrace{0 \cdots 0}_{N_{t}-q}]^{T} .
$$

The received signal $\mathbf{Y} \in \mathbb{C}^{N_{r} \times T}$ can be formulated as

$$
\mathbf{Y}=\mathbf{H X}^{i}+\mathbf{N} \text {, }
$$

where $\mathbf{H} \in \mathbb{C}^{N_{r} \times N_{t}}$ denotes the channel matrix, and $\mathbf{n} \in$ $\mathbb{C}^{N_{r} \times T}$ is the noise matrix, whose elements follow the Gaussian distribution as $\mathcal{C N}\left(0, \sigma^{2}\right)$.

At the receiver, assume that the estimated channel matrix is given by

$$
\hat{\mathbf{H}}=\mathbf{H}+\mathbf{H}_{e},
$$

where $\mathbf{H}_{e} \in \mathcal{C} \mathcal{N}\left(0, \sigma_{e}^{2}\right)$ and there is no channel estimation error for the case of $\sigma_{e}^{2}=0$. The ML detector is expressed as

$$
\hat{\mathbf{X}}=\underset{\mathbf{X} \in \mathbb{X}}{\arg \min }\|\mathbf{Y}-\hat{\mathbf{H} X}\|^{2},
$$

where $\mathbb{X}$ is the set of GIM symbols. 
B. ABEP analysis of GIM systems over generalized fading channel

Assuming that $\mathbf{X}^{i}$ and $\mathbf{X}^{j}$ are two different GIM symbols, the ABEP upper bound is expressed as

$$
P_{b}=\frac{1}{B 2^{B}} \sum_{i=1}^{2^{B}} \sum_{j \neq i}^{2^{B}} d\left(\mathbf{X}^{i}, \mathbf{X}^{j}\right) P\left(\mathbf{X}^{i} \rightarrow \mathbf{X}^{j}\right)
$$

where $d\left(\mathbf{X}^{i}, \mathbf{X}^{j}\right)$ denotes the HD between $\mathbf{X}^{i}$ and $\mathbf{X}^{j}$, $P\left(\mathbf{X}^{i} \rightarrow \mathbf{X}^{j}\right)$ denotes the pairwise error probability (PEP) event, which can be obtained by

$$
\begin{aligned}
& P\left(\mathbf{X}^{i} \rightarrow \mathbf{X}^{j} \mid \hat{\mathbf{H}}\right) \\
& =P\left(\left\|\mathbf{Y}-\hat{\mathbf{H}} \mathbf{X}^{i}\right\|^{2} \geq\left\|\mathbf{Y}-\hat{\mathbf{H}} \mathbf{X}^{j}\right\|^{2}\right) \\
& =P\left(\left\|\mathbf{N}-\mathbf{H}_{e} \mathbf{X}^{i}\right\|^{2} \geq\left\|\mathbf{H}\left(\mathbf{X}^{i}-\mathbf{X}^{j}\right)+\mathbf{N}-\mathbf{H}_{e} \mathbf{X}^{j}\right\|^{2}\right) \\
& \approx Q\left(\sqrt{\frac{\left\|\mathbf{H}\left(\mathbf{X}^{i}-\mathbf{X}^{j}\right)\right\|^{2}}{2\left(\sigma^{2}+\left\|\mathbf{X}^{i}\right\|^{2} \sigma_{e}^{2}\right)}}\right)
\end{aligned}
$$

where $Q(x)=\frac{1}{\pi} \int_{0}^{\frac{\pi}{2}} \exp \left(-\frac{x^{2}}{2 \sin \theta^{2}}\right) d_{\theta}$. Assuming that $\Delta=$ $\left(\mathbf{X}^{i}-\mathbf{X}^{j}\right)$ and $\gamma=\left\|\mathbf{H}\left(\mathbf{X}^{i}-\mathbf{X}^{j}\right)\right\|^{2}$, the PEP event can be obtained as

$$
\begin{aligned}
& P\left(\mathbf{X}^{i} \rightarrow \mathbf{X}^{j}\right)=\frac{1}{\pi} \int_{0}^{\frac{\pi}{2}} \int_{\gamma} \exp \left(-\frac{\gamma}{4\left(\sigma^{2}+\left\|\mathbf{X}^{i}\right\|^{2} \sigma_{e}^{2}\right) \sin ^{2} \theta}\right) f_{\gamma}(\gamma) d_{\gamma} d_{\theta} \\
& =\frac{1}{\pi} \int_{0}^{\frac{\pi}{2}} M_{\gamma}\left(-\frac{1}{4\left(\sigma^{2}+\left\|\mathbf{X}^{i}\right\|^{2} \sigma_{e}^{2}\right) \sin ^{2} \theta}\right) d_{\theta},
\end{aligned}
$$

where $M_{\gamma}(s)=\int_{\gamma} e^{s} f_{\gamma}(\gamma) d_{\gamma}$ is the MGF of $\gamma$.

According to [46], we have

$$
\begin{aligned}
& \gamma=\left\|\mathbf{H}\left(\mathbf{X}^{i}-\mathbf{X}^{j}\right)\right\|^{2} \\
& =\underbrace{\operatorname{vec}\left(\mathbf{H}^{H}\right)^{H}}_{\mathbf{u}} \underbrace{\left(\mathbf{I}_{N_{r}} \otimes \Delta \Delta^{H}\right)}_{\mathbf{B}} \underbrace{\operatorname{vec}\left(\mathbf{H}^{H}\right)}_{\mathbf{u}} \\
& =\mathbf{u}^{H} \mathbf{B u} .
\end{aligned}
$$

Based on [47], the MGF of $\gamma$ is given by

$$
M_{\gamma}(s)=\frac{\exp \left(-\overline{\mathbf{u}}^{H}\left(\mathbf{R}_{\mathbf{u}}\right)^{-1}\left(\mathbf{I}-\left(\mathbf{I}-s \mathbf{R}_{\mathbf{u}} \mathbf{B}\right)^{-1}\right) \overline{\mathbf{u}}\right)}{\left|\mathbf{I}-s \mathbf{R}_{\mathbf{u}} \mathbf{B}\right|},
$$

where $\overline{\mathbf{u}}=\mathbf{E}(\mathbf{u}), \mathbf{R}_{\mathbf{u}}=\mathbf{E}\left(\mathbf{u u}^{H}\right)$.

Then the PEP can be obtained by

$$
\begin{aligned}
& P\left(\mathbf{X}^{i} \rightarrow \mathbf{X}^{j}\right)=\frac{1}{\pi} \int_{0}^{\frac{\pi}{2}} M_{\gamma}\left(-\frac{1}{4\left(\sigma^{2}+\left\|\mathbf{X}^{i}\right\|^{2} \sigma_{e}^{2}\right) \sin ^{2} \theta}\right) d_{\theta} \\
& =\frac{1}{\pi} \int_{0}^{\frac{\pi}{2}} \frac{\exp \left(-\overline{\mathbf{u}}^{H}\left(\mathbf{R}_{\mathbf{u}}\right)^{-1}\left(\mathbf{I}-\left(\mathbf{I}+\frac{\mathbf{R}_{\mathbf{u}} \mathbf{B}}{4\left(\sigma^{2}+\left\|\mathbf{X}^{i}\right\|^{2} \sigma_{e}^{2}\right) \sin ^{2} \theta}\right)^{-1}\right) \overline{\mathbf{u}}\right)}{\left|\mathbf{I}+\frac{\mathbf{R} \mathbf{u} B}{4\left(\sigma^{2}+\left\|\mathbf{X}^{i}\right\|^{2} \sigma_{e}^{2}\right) \sin ^{2} \theta}\right|} d_{\theta} .
\end{aligned}
$$

To further simplify the calculation of (11), we use the characteristic function in (7) by [36]

$$
Q(x) \approx \frac{1}{12} e^{-\frac{x^{2}}{2}}+\frac{1}{4} e^{-\frac{2 x^{2}}{3}} .
$$

Then, the value of $P\left(\mathbf{X}^{i} \rightarrow \mathbf{X}^{j}\right)$ can be approximately expressed by

$$
\begin{aligned}
& P\left(\mathbf{X}^{i} \rightarrow \mathbf{X}^{j}\right) \\
& \approx \int\left(\frac{1}{12} e^{-\frac{\gamma}{4\left(\sigma^{2}+\left\|\mathbf{X}^{i}\right\|^{2} \sigma_{e}^{2}\right)}}+\frac{1}{4} e^{-\frac{\gamma}{3\left(\sigma^{2}+\left\|\mathbf{X}^{i}\right\|^{2} \sigma_{e}^{2}\right)}}\right) f_{\gamma}(\gamma) d \gamma \\
& =\frac{1}{12} M_{\gamma}\left(-\frac{1}{4\left(\sigma^{2}+\left\|\mathbf{X}^{i}\right\|^{2} \sigma_{e}^{2}\right)}\right)+\frac{1}{4} M_{\gamma}\left(-\frac{1}{3\left(\sigma^{2}+\left\|\mathbf{X}^{i}\right\|^{2} \sigma_{e}^{2}\right)}\right) \\
& =\frac{1}{12} \frac{\exp \left(-\overline{\mathbf{u}}^{H}\left(\mathbf{R}_{\mathbf{u}}\right)^{-1}\left(\mathbf{I}-\left(\mathbf{I}+\frac{\mathbf{R}_{\mathbf{u}} \mathbf{B}}{4\left(\sigma^{2}+\left\|\mathbf{X}^{i}\right\|^{2} \sigma_{\sigma}^{2}\right) \sin ^{2} \theta}\right)^{-1}\right) \overline{\mathbf{u}}\right)}{\left|\mathbf{I}+\frac{\mathbf{R}_{\mathbf{u}} \mathbf{B}}{4\left(\sigma^{2}+\left\|\mathbf{X}^{i}\right\|^{2} \sigma_{e}^{2}\right) \sin ^{2} \theta}\right|} \\
& +\frac{1}{4} \frac{\exp \left(-\overline{\mathbf{u}}^{H}\left(\mathbf{R}_{\mathbf{u}}\right)^{-1}\left(\mathbf{I}-\left(\mathbf{I}+\frac{\mathbf{R u \mathbf { u }}^{2}}{3\left(\sigma^{2}+\left\|\mathbf{X}^{i}\right\|^{2} \sigma_{e}^{2}\right) \sin ^{2} \theta}\right)^{-1}\right) \overline{\mathbf{u}}\right)}{\left|\mathbf{I}+\frac{\mathbf{R} \mathbf{B}}{3\left(\sigma^{2}+\left\|\mathbf{X}^{i}\right\|^{2} \sigma_{e}^{2}\right) \sin ^{2} \theta}\right|} .
\end{aligned}
$$

Hence, the ABEP is mainly associated with the value of $\overline{\mathbf{u}}$,
$\mathbf{R}_{\mathbf{u}}, \Delta=\left(\mathbf{X}^{i}-\mathbf{X}^{j}\right)$ and $d\left(\mathbf{X}^{i}, \mathbf{X}^{j}\right)$.

Since the values of $\overline{\mathbf{u}}, \mathbf{R}_{\mathbf{u}}$ are associated with the specific channel, which are calculated in Appendix, the ABEP can be further improved by optimizing the value of $d\left(\mathbf{X}^{i}, \mathbf{X}^{j}\right)$ and $\Delta=\left(\mathbf{X}^{i}-\mathbf{X}^{j}\right)$ for a given channel model.

\section{Bit-to-IC mapping optimization}

For a GIM system with $N_{t}$ elements and $N_{u}$ activated ones, there are a total of $C_{N_{\text {all }}}^{N}=\frac{N_{\text {all }} !}{N !\left(N_{\text {all }}-N\right) !}$ possible IC sets $\mathbb{I}_{q}$, $q=\left(1, \ldots, C_{N_{\text {all }}}^{N}\right)$. In the conventional GIM system, a specific IC set is selected randomly from $\left\{\mathbb{I}_{1}, \ldots, \mathbb{I}_{C_{N_{\text {all }}}^{N}}\right\}$. For each selected IC set $\mathbb{I}_{q}$, there are $N$ ! ways for the bit-to-IC mapping, resulting in different ABEPs. In this paper, we mainly focus on the optimization of the bit-to-IC mapping without APM symbol, i.e., select one IC and one mapping principle to obtain the smallest ABEP of $M=1$ as

$$
\text { s.t. } \forall \mathbb{I}_{q}, \mathbb{M}_{q} \text {, }
$$

where $\mathbb{M}_{q}$ is the bit-to-IC mapping set of the select IC set $\mathbb{I}_{q}$. To satisfy (14), one out of $C_{N_{\text {all }}}^{N} N !=\frac{N_{\text {all }} !}{\left(N_{\text {all }}-N\right) !}$ mapping principles should be selected, which becomes impractical for a relatively large value of $N_{\text {all }}$.

Specifically, for the case of $M=1$, the activated IC only transmits symbol ' 1 ', so that the ABEP can be represented as

$$
\begin{aligned}
& P_{b}=\frac{1}{B 2^{B}} \sum_{i=1}^{2^{B}} \sum_{j \neq i}^{2^{B}} d\left(\mathbf{X}^{i}, \mathbf{X}^{j}\right) P\left(\mathbf{X}^{i} \rightarrow \mathbf{X}^{j}\right) \\
& =\underbrace{\frac{1}{B 2^{B}} \sum_{i=1}^{2^{B}} \sum_{j \neq i}^{2^{B}} d\left(\mathbf{X}^{i}, \mathbf{X}^{j}\right) P\left(\mathbf{X}^{i} \rightarrow \mathbf{X}^{j} \mid n_{\mathrm{err}}=1\right)}_{P_{b}^{1}}+\cdots+ \\
& \underbrace{\frac{1}{B 2^{B}} \sum_{i=1}^{2^{B}} \sum_{j \neq i}^{2^{B}} d\left(\mathbf{X}^{i}, \mathbf{X}^{j}\right) P\left(\mathbf{X}^{i} \rightarrow \mathbf{X}^{j} \mid n_{\mathrm{err}}=N_{u}\right)}_{P_{b}^{N_{u}}},
\end{aligned}
$$

where $P_{b}^{n_{\text {err }}} n_{\text {err }}=1, \ldots, N_{u}$ denotes the ABEP of the PEP event with $n_{\text {err }}$ erroneous indices. According to (1)-(13), we usually have

$P\left(\mathbf{X}^{i} \rightarrow \mathbf{X}^{j} \mid n_{\mathrm{err}}=1\right) \gg \cdots \gg P\left(\mathbf{X}^{i} \rightarrow \mathbf{X}^{j} \mid n_{\mathrm{err}}=N_{u}\right)$.

Therefore, the optimization can be decomposed into $N_{u}$ steps as follows.

Step 1: Find the IC set $\mathcal{I}^{1}$ and the mapping set $\mathcal{M}^{1}$ by $\left(\mathcal{I}^{1}, \mathcal{M}^{1}\right)=\min _{\forall \mathbb{I}_{q}, \mathbb{M}_{q}} P_{b}^{1}$.

Step 2: Find the IC and the mapping set $\left(\mathcal{I}^{2}, \mathcal{M}^{2}\right)$ from $\left(\mathcal{I}^{1}, \mathcal{M}^{1}\right)$ by $\left(\mathcal{I}^{2}, \mathcal{M}^{2}\right)=\min _{\forall \mathcal{I}^{1}, \mathcal{M}^{1}} P_{b}^{2}$.

...

Step $N_{u}$ : Find the IC set and the mapping set $\left(\mathcal{I}^{N_{u}}, \mathcal{M}^{N_{u}}\right)$ from $\left(\mathcal{I}^{N_{u}-1}, \mathcal{M}^{N_{u}-1}\right)$ by $\left(\mathcal{I}^{N_{u}}, \mathcal{M}^{N_{u}}\right)=$ $\min _{\forall \mathcal{I}^{N_{u}-1}, \mathcal{M}^{N_{u}-1}} P_{b}^{N_{u}}$.

Since the value of $P\left(\mathbf{X}^{i} \rightarrow \mathbf{X}^{j} \mid n_{\mathrm{err}}\right)$ is independent of the bit-to-IC mapping in some GIM systems, the optimization is 


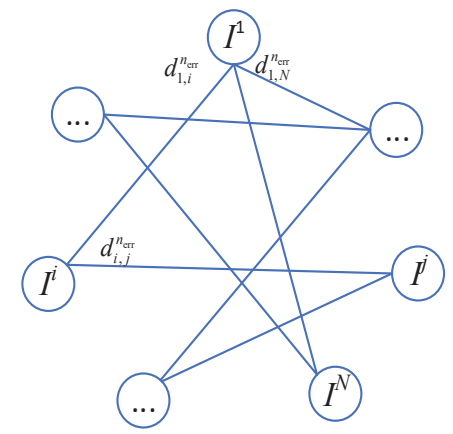

Fig. 2. A weighted graph $G_{n_{\mathrm{err}}}^{w}(V, E)$ for a specific IC set.

finally represented as

$$
\begin{aligned}
& \left(\mathcal{I}^{1}, \mathcal{M}^{1}\right) \approx \min _{\forall \mathbb{I}_{q}, \mathbb{M}_{q}} \sum_{i=1}^{2^{B}} \sum_{j \neq i}^{2^{B}} d\left(\mathbf{X}^{i}, \mathbf{X}^{j} \mid n_{\mathrm{err}}=1\right) \\
& \left(\mathcal{I}^{2}, \mathcal{M}^{2}\right)=\min _{\forall \mathcal{I}^{1}, \mathcal{M}^{1}} \sum_{i=1}^{2^{B}} \sum_{j \neq i}^{2^{B}} d\left(\mathbf{X}^{i}, \mathbf{X}^{j} \mid n_{\mathrm{err}}=2\right) \\
& \vdots \\
& \left(\mathcal{I}^{N_{u}}, \mathcal{M}^{N_{u}}\right)=\min _{\forall \mathcal{I}^{N_{u}-1}, \mathcal{M}^{N_{u}-1}} \sum_{i=1}^{2^{B}} \sum_{j \neq i}^{2^{B}} d\left(\mathbf{X}^{i}, \mathbf{X}^{j} \mid n_{\mathrm{err}}=N_{u}\right),
\end{aligned}
$$

where $d_{i j}^{n_{\text {err }}}=d\left(\mathbf{X}^{i}, \mathbf{X}^{j} \mid n_{\text {err }}\right) \in \mathbb{D}$ denotes the HD of the PEP event with $n_{\text {err }}$ erroneous indices, where the HD set $\mathbb{D}$ is

$$
\mathbb{D}=(\underbrace{1, \ldots, 1}_{C_{B}^{1}}, \underbrace{2, \ldots, 2}_{C_{B}^{2}}, \ldots, \underbrace{u, \ldots, u}_{C_{B}^{u}}, \ldots, \underbrace{B}_{C_{B}^{B}}) .
$$

To simplify the optimization, the above formulations will be transformed to graph theory based problems and lowcomplexity search algorithms will be designed.

\section{GRAPH THEORY BASED PROBLEM FORMULATION}

\section{A. Definitions of graphs}

A graph $G=(V(G), E(G))$ consists of two finite sets, where $V(G)$ denotes the vertex set of graph with $|V(G)|=n$ and $E(G)$ denotes the edge set of graph with $|E(G)|=m$. Each edge $e$ in $E$ is assigned as an unordered pair of vertices $(u, v)$, which is called the end vertices of $e$.

Incidence: When a vertex $v_{i}$ is an end vertex of some edge $e_{j}, v_{i}$ and $e_{j}$ are said to be incident with each other.

Adjacent: Two vertices are said to be adjacent if they are the end vertices of the same edge.

Degree: Let $v$ be a vertex of the graph $\mathrm{G}$. The degree $d(v)$ of $v$ is the number of edges of $G$ incident with $v$ by counting each self-loop twice. $\delta(G)$ and $\Delta(G)$ represent the minimum degree and maximum degree of $G$, respectively.

Subgraph: Let $G_{1}$ be a graph with vertex set $V\left(G_{1}\right)$ and edge set $E\left(G_{1}\right)$. If $G_{1}=\left(V\left(G_{1}\right), E\left(G_{1}\right)\right)$ is a subgraph of $G=(V(G), E(G))$, we have $V\left(G_{1}\right) \subset V(G), E\left(G_{1}\right) \subset$ $E(G)$.

Weighted graph: Let $G^{w}(V, E)$ be a weighted graph, where each edge $e$ has been assigned a weight $w(e)$.

Completed graph: If $G_{c}(V, E)$ is a completed graph with $V=\left(v_{1}, \ldots, v_{n}\right)$, we have $d\left(v_{1}\right)=d\left(v_{2}\right)=, \ldots,=d\left(v_{n}\right)=$ $n-1$.

\section{B. Problem formulation}

In this subsection, we reformulate the optimization of (17) into the formulation associated with a graph. Specifically, Fig. 2 presents a weighted graph $G_{n_{\text {err }}}(V, E)$ for a specific IC set of $V=\left(I^{1}, \ldots, I^{N}\right)$, where $I^{i}$ and $I^{j}$ are adjacent if there are $n_{\text {err }}$ different elements between them, and the weight of this edge is $w_{i, j}=d_{i j}^{n_{\text {err }}}$.

For the weighted graph $G_{n_{\text {err }}}^{w}(V, E)$ shown in Fig. 2, the corresponding weighted adjacent matrix is expressed as

$$
\mathbf{A}_{n_{\text {err }}}=\begin{gathered}
I^{1} \\
\vdots \\
I^{j} \\
\vdots \\
I^{N}
\end{gathered}\left[\begin{array}{cccccc}
0 & \cdots & d_{1, i}^{n_{\text {err }}} & \cdots & d_{1, N}^{n_{\text {err }}} \\
\vdots & \ddots & \vdots & \ddots & \vdots \\
0 & \cdots & d_{j, i}^{n_{\text {err }}} & \cdots & 0 \\
\vdots & \ddots & \vdots & \ddots & \vdots \\
d_{N, 1}^{n_{\text {err }}} & \cdots & 0 & \cdots & 0
\end{array}\right],
$$

where the $i$-th row and $j$-th column $A_{n_{\text {nor }}}^{i, j}$ is expressed as

$$
A_{n_{\text {err }}}^{i, j}=A_{n_{\text {err }}}^{j, i}=\left\{\begin{array}{l}
d_{i, j}^{n_{\text {err }}}, \text { if length }\left(\operatorname{setdiff}\left(I^{i}, I^{j}\right)\right)=n_{\text {err }} \\
0, \quad \text { else }
\end{array},\right.
$$

where $\operatorname{setdiff}(\mathbf{x}, \mathbf{y})$ is a function returning the different values between $\mathbf{x}$ and $\mathbf{y}$ and length $(\mathbf{x})$ is a function returning the length of $\mathbf{x}$. Hence, (17) can be finally represented as

$$
\begin{aligned}
& \left(\mathcal{I}^{1}, \mathcal{M}^{1}\right) \approx \min _{\forall \mathbb{I}_{q}, \mathbb{M}_{q}} W\left(\mathbf{A}_{1}\right), \\
& \left(\mathcal{I}^{2}, \mathcal{M}^{2}\right)=\min _{\forall \mathcal{I}^{1}, \mathcal{M}^{1}} W\left(\mathbf{A}_{2}\right), \\
& \vdots \\
& \left(\mathcal{I}^{N_{u}}, \mathcal{M}^{N_{u}}\right)=\min _{\forall \mathcal{I}^{N_{u}-1}, \mathcal{M}^{N_{u}-1}} W\left(\mathbf{A}_{N_{u}}\right),
\end{aligned}
$$

where $W\left(\mathbf{A}_{n_{\mathrm{err}}}\right)=\sum_{i=1}^{2^{B}} \sum_{j=1}^{2^{B}} \mathrm{~A}_{n_{\mathrm{err}}}^{i, j}$.

Based on the above analysis, we mainly aim to find the set $\left(\mathcal{I}^{1}, \mathcal{M}^{1}\right)$ in this paper. According to (21), it consists of two steps: 1) find the set $\mathcal{I}^{1}$; 2) find the set $\mathcal{M}^{1}$. Assuming that the graph $G_{1}^{a}(V, E)$ denotes a graph with $N_{\text {all }}$ vertexes as $V\left(G_{1}^{a}\right)=\left(I^{1}, I^{2}, \ldots, I^{N_{\text {all }}}\right)$, where $I^{i}$ and $I^{j}$ are adjacent if there are $n_{\text {err }}=1$ different elements. Then, finding the optimal IC set $\mathcal{I}^{1}$ can be reformulated as problem formulation 1 .

Problem formulation 1: Find a subgraph $G_{1}(V, E)$ from $G_{1}^{a}(V, E)$ with the minimum degree $\delta\left(G_{1}\right)$.

For a subgraph $G_{1}(V, E)$ with $V\left(G_{1}\right)=\left(I^{1}, \ldots, I^{N}\right), N$ blocks of information bits are mapped to this graph, resulting in $N$ ! kinds of weighted graphs. We should find one mapping principle to have the minimum weight. Since $N$ blocks of information bits consist of one weighted completed graph $G_{c}^{w}(V, E)$, where the weight between two adjacent vertexes is the HD between the corresponding information bits. Then, finding the optimal mapping set $\mathcal{M}^{1}$ can be transformed into problem formulation 2 .

Problem formulation 2: Find a subgraph $G_{1}^{w}(V, E)$ from the weighted complete graph $G_{c}^{w}(V, E)$ with the minimum weight, where $G_{1}^{w}(V, E)$ and $G_{1}(V, E)$ have the same vertex set and edge set.

\section{GRAPH THEORY ASSISTED IC SELECTION}

In this section, we mainly focus on solving the problem formulation 1 in Section III-B. As introduced in Section IIIB, we have to remove $N_{\text {re }}=N_{\text {all }}-N$ vertexes from the graph 


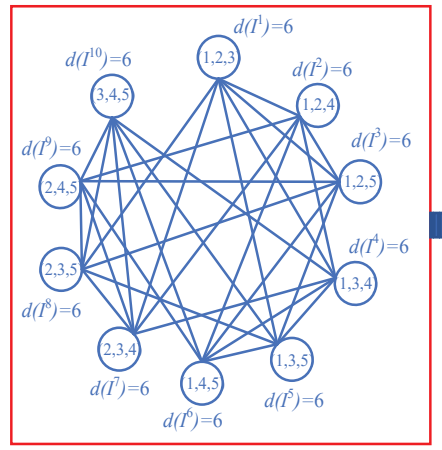

Step 1: Obtain the initial graph

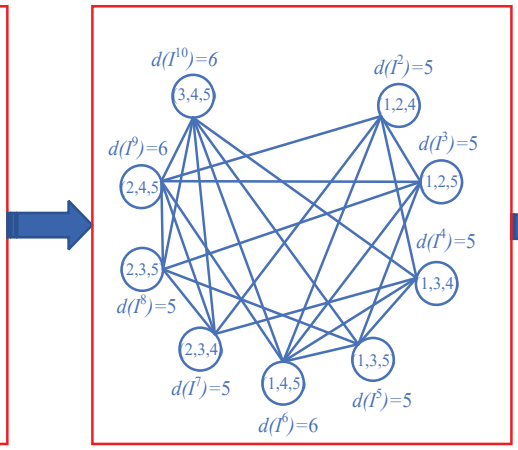

Step 2: Remove any one vertex with the maximum degree

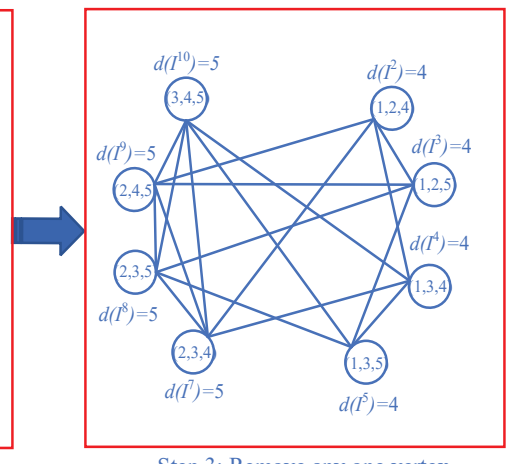

Step 3: Remove any one vertex with the maximum degree

Fig. 3. The proposed MDF algorithm for IC selection having $N_{t}=5$ and $N_{u}=3$.

$G_{1}^{a}(V, E)$. There are a total of $C_{N_{\text {all }}}^{N_{\text {re }}}$ possible IC graphs, resulting in different degrees. To find the graph with the minimum degree, the optimal algorithm has to calculate the degrees of $C_{N_{\text {all }}}^{N_{\text {re }}}$ graphs, which becomes prohibitively complicated and challenging. In this section, we propose a low-complexity MDF algorithm to address the above issue. Specifically, the IC selection consists of $N_{\text {re }}$ steps. For each step, the vertex with the maximum degree is removed, and the degree of remaining vertexes is updated. Repeat the above processes until we remove $N_{\mathrm{re}}$ vertexes. The complexity order of the MDF algorithm is $O\left(N_{\mathrm{re}} N\right)$, which becomes straightforward for any setup of $N_{t}$ and $N_{u}$. For easy understanding, we present an example of the IC selection as follows.

\section{A. MDF assisted IC selection for $N_{t}=5$ and $N u=3$}

Fig. 3 presents the MDF assisted IC selection for $N_{t}=5$ and $N u=3$. As shown in Fig. 2, the initial graph $G_{1}^{a}(V, E)$ has 10 vertexes as

$$
\begin{aligned}
& V\left(G_{1}^{a}\right)=[\underbrace{(1,2,3)}_{I^{1}}, \underbrace{(1,2,4)}_{I^{2}}, \underbrace{(1,2,5)}_{I^{3}}, \underbrace{(1,3,4)}_{I^{4}}, \underbrace{(1,3,5)}_{I^{5}} \\
& \underbrace{(1,4,5)}_{I^{6}}, \underbrace{(2,3,4)}_{I^{7}}, \underbrace{(2,3,5)}_{I^{8}}, \underbrace{(2,4,5)}_{I^{9}}, \underbrace{(3,4,5)}_{I^{10}}] .
\end{aligned}
$$

We have to remove $N_{\text {re }}=2$ ICs for the bit-to-IC mapping.

Step 1: Remove the first vertex with the maximum degree. As shown in Fig. 3, we have $d\left(I^{1}\right)=\ldots=d\left(I^{10}\right)=6$ at the beginning, so that any vertex is suitable for removal. Taking removing the vertex $I^{1}$ for example, we update the degree of each vertex as

$$
\begin{aligned}
& d\left(I^{2}\right)=d\left(I^{3}\right)=d\left(I^{4}\right)=d\left(I^{5}\right)=d\left(I^{7}\right)=d\left(I^{8}\right)=5, \\
& d\left(I^{6}\right)=d\left(I^{9}\right)=d\left(I^{10}\right)=6 .
\end{aligned}
$$

Step 2: Remove the second vertex with the maximum degree. Taking removing the vertex $I^{6}$ for example, we update the degree of each vertex as

$$
\begin{aligned}
& d\left(I^{2}\right)=d\left(I^{3}\right)=d\left(I^{4}\right)=d\left(I^{5}\right)=4, \\
& d\left(I^{7}\right)=d\left(I^{8}\right)=d\left(I^{9}\right)=d\left(I^{10}\right)=5 .
\end{aligned}
$$

As a result, an optimal IC with the minimum degree is $\mathbb{I}_{o}=$ $\left(I^{2}, I^{3}, I^{4}, I^{5}, I^{7}, I^{8}, I^{9}, I^{10}\right)$.

\section{B. MDF assisted generalized IC selection}

For a generalized IC selection, we have to remove $N_{\text {re }}$ vertexes from $\left\{I^{1}, \ldots, I^{N_{\text {all }}}\right\}$, so that the IC selection consists of $N_{\text {re }}$ steps. Firstly, we obtain the initial graph $G_{1}^{a, 0}(V, E)$ including $N_{\text {all }}$ vertexes. Assuming that $\mathcal{A}^{0}\left(I^{i}\right)$ denotes the adjacent vertex set of $I^{i}$, we have

$$
\left.d^{0}\left(I^{i}\right)=\left|\mathcal{A}^{0}\left(I^{i}\right)\right|=C_{N_{u}}^{N_{u}-1}\left(N_{t}-N_{u}\right), I^{i} \in V\left(G_{1}^{a, 0}\right)\right) .
$$

Step $\mathbf{t}\left(t=1, \ldots, N_{\text {re }}\right)$ : Remove the $l$-th vertex with the maximum degree based on the graph $V\left(G_{1}^{a, t-1}\right)$. Then, update the adjacent vertex set $\mathcal{A}^{t}\left(I^{i}\right)=\mathcal{A}^{t-1}\left(I^{i}\right) \backslash I^{l}$. The degree of the remaining vertex can be updated as $d^{t}\left(I^{i}\right)=\left|\mathcal{A}^{t}\left(I^{i}\right)\right|$. Repeat the above operations $N_{\text {re }}$ times, so that the complexity order of the proposed MDF algorithm is $O\left(N_{\mathrm{re}} N\right)$.

\section{Proof of the proposed MDF algorithm}

In this subsection, we will demonstrate that the proposed MDF algorithm is identical as the optimal one using the mathematical induction technique. The proposed MDF algorithm consists of $N_{\text {re }}$ steps. As shown in Section IV-B, the degree of each vertex becomes different from step 2. Assuming that $\mathbf{d}^{1}=\left(d^{1}\left(I^{1}\right), \ldots, d^{1}\left(I^{N_{\text {all }}-1}\right)\right)$, we have $\delta^{1}=\sum \mathbf{d}^{1}$ and the proof is given as follows.

1) In the $t=2$-th step, assuming that $d^{1}\left(I^{q}\right)=\max \left(\mathbf{d}^{1}\right)$, we have $d^{1}\left(I^{q}\right) \geq d^{1}\left(I^{p}\right) p \neq q$. Then, we obtain two different graphs $H_{p}\left(V^{2}, E^{2}\right)$ and $H_{q}\left(V^{2}, E^{2}\right)$ by removing the $p$-th vertex or the $q$-th vertex. Assuming that the total degrees of $H_{p}\left(V^{2}, E^{2}\right)$ and $H_{q}\left(V^{2}, E^{2}\right)$ are $\delta^{2, p}$ and $\delta^{2, q}$, it is straightforward to have

$$
\delta^{2, p}=\delta^{1}-d^{1}\left(I^{p}\right), \delta^{2, q}=\delta^{1}-d^{1}\left(I^{q}\right) .
$$

It is evident that $\delta^{2, q}<\delta^{2, p}$.

2) In the $t=K$-th step, $\delta^{K}=\sum_{i=1}^{N_{\text {all }}-K} d^{K}\left(I^{q}\right)$ has the smallest value. Assuming that $d^{K}\left(I^{q}\right)=\max \left(\mathbf{d}^{K}\right)$, we have $d^{K}\left(I^{q}\right) \geq d^{K}\left(I^{p}\right) p \neq q$. In the $t=(K+1)$ th step, by removing the $q$-th or $p$-th vertex, we obtain $\delta^{K+1, q}=\delta^{K}-d^{K}\left(I^{q}\right)$ and $\delta^{K+1, p}=\delta^{K}-d^{K}\left(I^{p}\right)$. It is easy to obtain that $\delta^{K+1, q} \leq \delta^{K+1, p}$.

3 ) It is concluded from the above two steps that the proposed MDF algorithm always has the minimum degree. 


\section{GRAPH THEORY ASSISTED BIT-TO-IC MAPPING}

In this section, we mainly focus on solving the problem formulation 2 of section III-B. For a specific IC graph, there are a total of $N$ ! mapping choices, resulting in $N$ ! weighted graphs. The aim of the bit-to-IC mapping is to find one weighted graph with the minimum weight. To tackle this issue, a MWF based low-complexity mapping principle is proposed. Specifically, the proposed algorithm consists of $N$ steps. In each step, one block of information bits are mapped to a specific IC index. We first decide which IC index should be mapped based on the adjacent matrix. Next, one block of information bits which make the current weighted graph to have the minimum weight will be mapped to this IC index. Repeat the above operations $N$ times. For easy understanding, we will introduce an example first and then present the generalized algorithm.

\section{A. Bit-to-IC mapping for $N_{t}=5, N_{u}=3$}

For the case of $N_{t}=5, N_{u}=3$, we reformulate the selected IC set as

$$
\begin{aligned}
& \mathbb{I}_{o}=[\underbrace{(1,2,4)}_{I^{1}}, \underbrace{(1,2,5)}_{I^{2}}, \underbrace{(1,3,4)}_{I^{3}}, \underbrace{(1,3,5)}_{I^{4}}, \\
& \underbrace{(2,3,4)}_{I^{5}}, \underbrace{(2,3,5)}_{I^{6}}, \underbrace{(2,4,5)}_{I^{7}}, \underbrace{(3,4,5)}_{I^{8}}] .
\end{aligned}
$$

Step 1: Obtain the adjacent matrix $\mathbf{A}_{1}$ as

$$
\begin{aligned}
& \begin{array}{llllllll}
I^{1} & I^{2} & I^{3} & I^{4} & I^{5} & I^{6} & I^{7} & I^{8}
\end{array} \\
& \mathbf{A}_{1}=\begin{array}{l}
I^{1} \\
I^{2} \\
I^{3} \\
I^{4} \\
I^{5} \\
I^{6} \\
I^{7} \\
I^{8}
\end{array}\left[\begin{array}{cccccccc}
0 & d_{12}^{1} & d_{13}^{1} & 0 & d_{15}^{1} & 0 & d_{17}^{1} & 0 \\
d_{21}^{1} & 0 & 0 & d_{24}^{1} & 0 & d_{26}^{1} & d_{27}^{1} & 0 \\
d_{31}^{1} & 0 & 0 & d_{34}^{1} & d_{35}^{1} & 0 & 0 & d_{38}^{1} \\
0 & d_{42}^{1} & d_{43}^{1} & 0 & 0 & d_{46}^{1} & 0 & d_{48}^{1} \\
d_{51}^{1} & 0 & d_{53}^{1} & 0 & 0 & d_{56}^{1} & d_{57}^{1} & d_{58}^{1} \\
0 & d_{62}^{1} & 0 & d_{64}^{1} & d_{65}^{1} & 0 & d_{67}^{1} & d_{68}^{1} \\
d_{71}^{1} & d_{72}^{1} & 0 & 0 & d_{75}^{1} & d_{76}^{1} & 0 & d_{78}^{1} \\
0 & 0 & d_{83}^{1} & d_{84}^{1} & d_{85}^{1} & d_{86}^{1} & d_{87}^{1} & 0
\end{array}\right] .
\end{aligned}
$$

Then, its corresponding nonzero index set can be expressed as

$$
\mathcal{A}=\left[\begin{array}{lllll}
2 & 3 & 5 & 7 & 0 \\
1 & 4 & 6 & 7 & 0 \\
1 & 4 & 5 & 8 & 0 \\
2 & 3 & 6 & 8 & 0 \\
1 & 3 & 6 & 7 & 8 \\
2 & 4 & 5 & 7 & 8 \\
1 & 2 & 5 & 6 & 8 \\
3 & 4 & 5 & 6 & 7
\end{array}\right],
$$

where the elements of the $i$-th row of $\mathcal{A}$ represent the IC indices with one different element with the IC $I^{i}$.

Step 2: The bit-to-IC mapping can begin at any IC vertex with any bits index as

$$
\begin{aligned}
& b_{1}=(000), b_{2}=(001), b_{3}=(010), b_{4}=(011), \\
& b_{5}=(100), b_{6}=(101), b_{7}=(110), b_{8}=(111) .
\end{aligned}
$$

Assuming that $\mathcal{M}_{n} n=1, \ldots, N$ denotes the $n$-th mapping vector, $V_{b}^{n}$ is the set of the nonzero elements of $\mathcal{M}_{n}$, and $V_{I}^{n}$ is the index set of these nonzero elements, we have $\mathcal{M}_{0}=$ $[0,0,0,0,0,0,0,0]$ and $V_{I}^{0}=[\phi] V_{b}^{0}=[\phi]$ at the beginning. Especially, $V_{b}^{n}$ and $V_{I}^{n}$ also present the bits index set and IC index set that have been mapped, respectively. Taking $b_{2} \rightarrow$ $I^{1}$ for example, we have $\mathcal{M}_{1}=[2,0,0,0,0,0,0,0], V_{I}^{1}=$ $[1], V_{b}^{1}=[2]$ and $\mathcal{M}_{\text {left }}=[1,3,4,5,6,7,8]$. Then, the next mapping process begins at the set $\mathcal{A}_{1}=[2,3,5,7]$. Taking $I^{2}$ for example, we have to find one bit index from $\mathcal{M}_{\text {left }}$ to have the minimum HD between $I^{1}$ and $I^{2}$. It is easy to obtain $b_{1} \rightarrow I^{2}$. Finally, update the mapping set as $\mathcal{M}_{2}=$ $[2,1,0,0,0,0,0,0], V_{I}^{2}=[1,2], V_{b}^{2}=[2,1]$ and $\mathcal{M}_{\text {left }}=$ $[3,4,5,6,7,8]$.

Step 3: Find the 3-rd IC index that has the largest number of adjacent vertexes with $V_{I}$ for mapping by

$$
\begin{aligned}
& l_{i}=\underset{\forall i}{\operatorname{length}}\left(\operatorname{intersect}\left(\mathcal{A}_{i}, V_{I}^{t-1}\right)\right), i \notin V_{I}^{t-1}, t=3, \ldots, N, \\
& i_{t}=\underset{\forall i \max }{\arg \max }\left(l_{i}\right)
\end{aligned}
$$

where intersect $(\mathbf{x}, \mathbf{y})$ is a function returning the same value between $\mathbf{x}$ and $\mathbf{y}$. According to (31), we have $\mathbf{l}^{3}=$ $\left[l_{1}, l_{2}, l_{3}, \ldots, l_{8}\right]=[0,0,1,1,1,1,2,0]$, and the mapping starts at $I^{7}$. Next, find one bit index from $\mathcal{M}_{\text {left }}$ to have the minimum HD with the mapped ICs $I^{1}$ and $I^{2}$. Specifically, we have

$$
\begin{aligned}
& \left(b_{3} \rightarrow I^{7}\right) \rightarrow d\left(I^{7}, I^{1}\right)+d\left(I^{7}, I^{2}\right)=d_{71}^{1}+d_{72}^{1}=3, \\
& \left(b_{4} \rightarrow I^{7}\right) \rightarrow d\left(I^{7}, I^{1}\right)+d\left(I^{7}, I^{2}\right)=d_{71}^{1}+d_{72}^{1}=3, \\
& \left(b_{5} \rightarrow I^{7}\right) \rightarrow d\left(I^{7}, I^{1}\right)+d\left(I^{7}, I^{2}\right)=d_{71}^{1}+d_{72}^{1}=3, \\
& \left(b_{6} \rightarrow I^{7}\right) \rightarrow d\left(I^{7}, I^{1}\right)+d\left(I^{7}, I^{2}\right)=d_{71}^{1}+d_{72}^{1}=3, \\
& \left(b_{7} \rightarrow I^{7}\right) \rightarrow d\left(I^{7}, I^{1}\right)+d\left(I^{7}, I^{2}\right)=d_{71}^{1}+d_{72}^{1}=5, \\
& \left(b_{8} \rightarrow I^{7}\right) \rightarrow d\left(I^{7}, I^{1}\right)+d\left(I^{7}, I^{2}\right)=d_{71}^{1}+d_{72}^{1}=5 .
\end{aligned}
$$

Hence, we have $I^{7} \in\left\{b_{3}, b_{4}, b_{5}, b_{6}\right\}$. Taking $b_{3} \rightarrow I^{7}$ for example, we update the mapping set $\mathcal{M}_{3}=[2,1,0,0,0,0,3,0]$, $V_{I}^{3}=[1,2,7], V_{b}^{3}=[2,1,3]$ and $\mathcal{M}_{\text {left }}=[4,5,6,7,8]$.

Step 4: Find the 4-th IC index for mapping using $V_{I}^{3}=$ $[1,2,7]$. According to $(31)$, we have $\mathbf{1}^{4}=\left[l_{1}, l_{2}, l_{3}, \ldots, l_{8}\right]=$ $[0,0,1,1,2,2,0,1]$, so that we can start at $I^{5}$. Since

$$
\begin{aligned}
& \left(b_{4} \rightarrow I^{5}\right) \rightarrow d\left(I^{5}, I^{1}\right)+d\left(I^{5}, I^{7}\right)=d_{51}^{1}+d_{52}^{1}=2, \\
& \left(b_{5} \rightarrow I^{5}\right) \rightarrow d\left(I^{5}, I^{1}\right)+d\left(I^{5}, I^{7}\right)=d_{51}^{1}+d_{52}^{1}=4, \\
& \left(b_{6} \rightarrow I^{5}\right) \rightarrow d\left(I^{5}, I^{1}\right)+d\left(I^{5}, I^{7}\right)=d_{51}^{1}+d_{52}^{1}=4, \\
& \left(b_{7} \rightarrow I^{5}\right) \rightarrow d\left(I^{5}, I^{1}\right)+d\left(I^{5}, I^{7}\right)=d_{51}^{1}+d_{52}^{1}=4, \\
& \left(b_{8} \rightarrow I^{5}\right) \rightarrow d\left(I^{5}, I^{1}\right)+d\left(I^{5}, I^{7}\right)=d_{51}^{1}+d_{52}^{1}=4,
\end{aligned}
$$

it is obvious that $b_{4} \rightarrow I^{5}$. The mapping set can be updated by $\mathcal{M}_{4}=[2,1,0,0,4,0,3,0], V_{I}^{4}=[1,2,5,7], V_{b}^{4}=[2,1,4,3]$ and $\mathcal{M}_{\text {left }}=[5,6,7,8]$.

Step 5: Find the 5-th IC index for mapping using $V_{I}^{4}=$ $[1,2,5,7]$. According to $(31)$, we have $\mathbf{1}^{5}=\left[l_{1}, l_{2}, l_{3}, \ldots, l_{8}\right]=$ $[0,0,2,1,0,3,0,2]$, so that we can start at $I^{6}$. Since

$$
\begin{aligned}
& \left(b_{5} \rightarrow I^{6}\right) \rightarrow d_{62}^{1}+d_{65}^{1}+d_{67}^{1}=6, \\
& \left(b_{6} \rightarrow I^{6}\right) \rightarrow d_{62}^{1}+d_{65}^{1}+d_{67}^{1}=7, \\
& \left(b_{7} \rightarrow I^{6}\right) \rightarrow d_{62}^{1}+d_{65}^{1}+d_{67}^{1}=5, \\
& \left(b_{8} \rightarrow I^{6}\right) \rightarrow d_{62}^{1}+d_{65}^{1}+d_{67}^{1}=6,
\end{aligned}
$$

it is easy to obtain $b_{7} \rightarrow I^{6}$. The mapping set is updated as $\mathcal{M}_{5}=[2,1,0,0,4,7,3,0], V_{I}^{5}=[1,2,5,6,7], V_{b}^{5}=$ $[2,1,4,7,3]$ and $\mathcal{M}_{\text {left }}=[5,6,8]$.

Step 6: The 6-th IC index should be mapped is $I^{8}$ via $\mathbf{l}^{6}=\left[l_{1}, l_{2}, l_{3}, \ldots, l_{8}\right]=[0,0,2,2,0,0,0,3]$. Since

$$
\begin{aligned}
& \left(b_{5} \rightarrow I^{8}\right) \rightarrow d_{85}+d_{86}+d_{87}=6, \\
& \left(b_{6} \rightarrow I^{8}\right) \rightarrow d_{85}+d_{86}+d_{87}=7, \\
& \left(b_{8} \rightarrow I^{8}\right) \rightarrow d_{85}+d_{86}+d_{87}=4,
\end{aligned}
$$

it is easy to obtain that $b_{8} \rightarrow I^{8}$. The mapping set can be updated by $\mathcal{M}_{6}=[2,1,0,0,4,7,3,8], V_{I}^{6}=[1,2,5,6,7,8]$, $V_{b}^{6}=[2,1,4,7,3,8]$ and $\mathcal{M}_{\text {left }}=[5,6]$.

Step 7: The 7-th IC index should be mapped is $I^{3}$ via 


$$
\begin{aligned}
\mathbf{l}^{7}=\left[l_{1}, l_{2}, l_{3}, \ldots, l_{8}\right]=[0,0,3,3,0,0,0,0] . \text { Since } \\
\left(b_{5} \rightarrow I^{3}\right) \stackrel{\rightarrow}{=} d_{31}+d_{35}+d_{38}=7, \\
\left(b_{6} \rightarrow I^{3}\right) \rightarrow d_{31}+d_{35}+d_{38}=4,
\end{aligned}
$$

it is obvious that $b_{6} \rightarrow I^{3}$. The mapping set is updated by $\mathcal{M}_{7}=[2,1,6,0,4,7,3,8], V_{I}^{7}=[1,2,3,5,6,7,8], V_{b}^{7}=$ $[2,1,6,4,7,3,8]$ and $\mathcal{M}_{\text {left }}=[5]$.

Step 8: Based on Step 7, the final mapping set is $\mathcal{M}_{8}=$ $[2,1,6,5,4,7,3,8]$ and $\delta(G)=48$.

\section{B. MWF based generalized bit-to-IC mapping}

In this subsection, a low complexity generalized bit-to-IC mapping is introduced as follows.

Step 1: Obtain the weighted adjacent matrix $\mathbf{A}_{1}$ and its corresponding adjacent vertex set $\mathcal{A}$ based on the obtained IC set. Then, initialize the mapping set, mapped IC index set, mapped bit index set and the bit index set as $\mathcal{M}_{0}=$ $[0,0,0,0,0,0,0,0], V_{I}^{0}=\phi, V_{b}^{0}=\phi$ and $\mathcal{M}_{b}=[1, \ldots, N]$, respectively.

Step 2: Map the first IC index. Taking $I^{1}=b_{n}$ for example, we can update the mapping sets as $\mathcal{M}_{1}=[n, 0,0,0,0,0,0,0]$, $V_{I}^{1}=[1], V_{b}^{1}=[n]$ and $\mathcal{M}_{\text {left }}=\mathcal{M}_{b} \backslash V_{b}^{1}$.

Step $\mathbf{t}+1$ : Map the $t$-th IC index. Assuming that $\mathcal{M}_{t-1}\left(V_{I}^{t-1}\right)=V_{b}^{t-1}$, the $t$-th IC index $i_{t}$ can be obtained via (31) using $\mathcal{A}$ and $V_{I}^{t-1}$. Then, we obtain the adjacent set of $I^{i_{t}}$ as

$$
V_{I}^{i_{t}}=\operatorname{intersect}\left(\mathcal{A}_{i_{t}}, V_{I}^{t-1}\right) .
$$

The bit index for $I^{i_{t}}$ is belong to

$$
Q_{1}=\min _{q \in M_{\text {left }}}\left(\sum_{j \in V_{I}^{i_{t}}} d_{i_{t}, j}^{1}\right) .
$$

Then, the mapping sets can be updated by $V_{I}^{t}=V_{I}^{t-1} \cup i_{t}$, $V_{b}^{t}=V_{b}^{t-1} \cup Q_{1}(1), \mathcal{M}_{t}\left(V_{I}^{t}\right)=V_{b}^{t}$ and $\mathcal{M}_{\text {left }}=\mathcal{M}_{b} \backslash V_{b}^{t}$.

Repeat Steps $(t+1)$ until all the IC indices are mapped.

Tables I and II present some mapping examples, where bpcu denotes the bits per channel use. It can be observed from Tables I and II that the value of $W\left(\mathbf{A}_{1}\right)$ with our proposed algorithm is substantial lower than that of conventional counterpart. We have checked that the proposed mapping principle is similar as the optimal one at low transmission rates (i.e. $\left.\left(N_{t}, N_{u}\right)=(4,2),(5,2),(6,2),(6,3)\right)$. For other setups, it is challenging to obtain the optimal mapping principle by calculating the weights of $C_{N_{\text {all }}}^{N} N$ ! weighted graphs. The complexity orders of different bit-to-IC mapping methods are presented in Table III and their comparisons for specific setups are presented in Table IV. As observed from Tables III and IV, it is obvious that the proposed method makes the bit-to-IC mapping optimization of high throughput practical. Moreover, by using the bit-to-IC mapping Tables of I and II, the end-toend complexity of the proposed method based GIM system is the same as the conventional counterpart.

\section{Simulation Results}

In this section, the performances of the GIM systems with our proposed bit-to-IC mapping principles are compared with the conventional counterparts using the bit-to-IC mapping

\begin{tabular}{|c|c|c|c|}
\hline Scheme & IC selection & Mapping & Total \\
\hline Conventional & $O(1)$ & $O(1)$ & $O(1)$ \\
\hline Optimal & \multicolumn{2}{|c|}{$O\left(C_{N_{\mathrm{all}}^{N}} N !\right)$} & $O\left(C_{N_{\mathrm{all}}^{N}} N !\right)$ \\
\hline Proposed & $O\left(N_{\mathrm{re}} N\right)$ & $O\left(N^{2}\right)$ & $O\left(N_{\mathrm{re}} N\right)+O\left(N^{2}\right)$ \\
\hline
\end{tabular}
Tables I and II. ML detectors are employed for all the GIM systems. The main difference between our proposed GIM
TABLE III

COMPLEXITY ORDER OF DIFFERENT BIT-TO-IC METHODS

COMPLEXITY COMPARISON OF THE SPECIFIC SETUPS

\begin{tabular}{|c|c|c|c|c|}
\hline$\left(N_{t}, N_{u}\right)$ & $(6,3)$ & $(8,3)$ & $(10,3)$ & $(12,3)$ \\
& $4 \mathrm{bpcu}$ & $5 \mathrm{bpcu}$ & $6 \mathrm{bpcu}$ & $7 \mathrm{bpcu}$ \\
\hline Optimal & $O\left(10^{17}\right)$ & $O\left(10^{51}\right)$ & $O\left(10^{123}\right)$ & $O\left(10^{279}\right)$ \\
\hline Proposed & $O(320)$ & $O(1792)$ & $O(7680)$ & $O\left(10^{4}\right)$ \\
\hline
\end{tabular}

system and the conventional one lies in the bit-to-IC mapping table used for transmission, which is shown in Tables I and II. Moreover, the analytical ABEP performances are added as benchmarkers.

\section{A. Performance comparison of GIM based MIMO systems}

Figs. 4 and 5 compare the performance of GSM system having $N_{t}=6, N_{u}=2, N_{r}=6, M=2$ over different channels. Specifically, Rayleigh fading channel with different channel estimated errors and correlated coefficients are employed in Fig. 4, while Rician channel with $K=5$ and Nakagami$m$ channel with $m=2$ and $m=3$ are employed in Fig. 5. The ABEP upper bound of Rayleigh fading channel with $\sigma_{e}^{2}=0, \rho_{t}=\rho_{r}=0$ and $\sigma_{e}^{2}=1 /\left(10^{S N R / 10}\right), \rho_{t}=\rho_{r}=0$ are calculated via (6) using (13) or (42), while that of Rayleigh fading channel with $\sigma_{e}^{2}=0, \rho_{t}=\rho_{r}=0.6$ is calculated via via (6) using (13) and (56). The ABEP upper bound of Rician channel is calculated via (6) using (49) and (13), while that of Nakagami- $m$ channel is obtained via (6) using (45) and (13). As observed from Figs. 4 and 5, the proposed bit-to-IC mapping based GSM system exhibits better performance than the conventional counterpart over different fading channels and imperfect CSI.

Next, Figs. 6-8 compare the performances of the GSM systems employing the proposed mapping approach to that of the conventional counterpart under different antenna configurations. $N_{r}=4$, Rayleigh flat fading with perfect CSI and ML detectors are employed for all the GSM systems. For simplicity, $\left(N t, N_{u}, M\right)$ represents the antenna setups in GSM systems. It is observed from Fig. 6(a) that the proposed mapping principle based GSM systems with $(8,2,1),(8,3,1)$, $(8,4,1)$ outperforms the conventional counterparts by $1 \mathrm{~dB}, 1$ $\mathrm{dB}$ and $0.6 \mathrm{~dB}$ at $\mathrm{BER}=10^{-4}$, respectively. When $M$ increases to two, they still provide $0.6 \mathrm{~dB}, 0.6 \mathrm{~dB}$ and $0.4 \mathrm{~dB}$ gains over conventional counterpart for $(8,2,2),(8,3,2),(8,4,2)$, respectively. It can be observed from Fig. 7 that the proposed mapping principle based GSM systems with $(10,2,1)$, $(10,3,1),(10,2,2),(10,3,2)$ outperforms the conventional counterpart by $0.6 \mathrm{~dB}, 1 \mathrm{~dB}, 0.4 \mathrm{~dB}$ and $0.55 \mathrm{~dB}$, respectively. It can be observed from Fig. 8 that the proposed mapping principle based GSM systems with $(12,3,1),(12,3,2),(16,2,1)$, $(16,2,2)$ outperforms the conventional counterpart by $0.9 \mathrm{~dB}$, $0.5 \mathrm{~dB}, 0.7 \mathrm{~dB}$ and $0.5 \mathrm{~dB}$, respectively.

Figs. 9 and 10 compare the performances of the MS-GSTSK systems employing the proposed mapping principle to that of 
TABLE I

BIT-TO-IC MAPPING FOR DIFFERENT GIM SCHEMES

\begin{tabular}{|c|c|c|c|c|c|c|}
\hline \multicolumn{2}{|c|}{ Scheme } & $\begin{array}{l}\text { bit-to-IC mapping } \\
\text { IC } \quad \text { set }: \mathbb{I} \rightarrow \text { bits index }\end{array}$ & $N_{\mathrm{re}}$ & $\delta(G)$ & $W\left(\mathbf{A}_{1}\right)$ & $\frac{W\left(\mathbf{A}_{1}\right)}{B 2^{B}}$ \\
\hline \multirow{2}{*}{$\begin{array}{l}N_{t}=6 \\
N_{u}=2\end{array}$} & Pro. & {$[(1,4),(1,5),(1,6),(2,6),(4,5),(2,5),(3,6),(2,3)] \rightarrow[1,2,3,4,5,6,7,8]$} & 2 & 28 & 36 & 1.5 \\
\hline & Con. & {$[(2,5),(3,4),(3,6),(4,5),(4,6),(5,6),(3,5),(2,6)] \rightarrow[1,2,3,4,5,6,7,8]$} & 2 & 38 & 76 & 3.17 \\
\hline \multirow{2}{*}{$\begin{array}{c}N_{t}=6 \\
N_{u}=3 \\
4 \text { bpcu }\end{array}$} & Pro. & $\begin{array}{l}(1,2,5),(1,2,4),(1,2,6),(1,4,6),(1,3,5),(1,3,4),(1,5,6),(1,3,6)] \rightarrow[1, \ldots, 8] \\
{[(2,3,5),(2,3,4),(2,5,6),(2,3,6),(2,4,5),(3,4,5),(4,5,6),(3,4,6)] \rightarrow[9, \ldots, 16]}\end{array}$ & 4 & 108 & 160 & 2.5 \\
\hline & Con. & $\begin{array}{l}{[(1,3,6),(1,4,5),(2,4,6),(2,5,6),(2,3,5),(3,4,6),(1,3,4),(3,5,6)] \rightarrow[1, \ldots, 8]} \\
{[(4,5,6),(1,3,5),(3,4,5),(2,3,4),(2,3,6),(2,4,5),(1,5,6),(1,4,6)] \rightarrow[9, \ldots, 16]}\end{array}$ & 4 & 120 & 304 & 4.75 \\
\hline \multirow{2}{*}{$\begin{array}{c}N_{t}=7 \\
N_{u}=2 \\
4 \mathrm{bpcu}\end{array}$} & Pro. & $\begin{array}{l}(1,3),(5,7),(1,6),(6,7),(3,5),(3,7),(3,6),(2,6)] \rightarrow[1, \ldots, 8] \\
{[(1,5),(4,7),(1,4),(2,7),(4,5),(2,5),(4,6),(2,4)] \rightarrow[9, \ldots, 16]}\end{array}$ & 5 & 116 & 188 & 2.94 \\
\hline & Con. & $\begin{array}{l}{[(3,7),(5,7),(2,4),(2,6),(3,6),(4,5),(1,7),(2,3)] \rightarrow[1, \ldots, 8]} \\
{[(3,4),(2,7),(5,6),(3,5),(2,5),(4,6),(6,7),(4,7)] \rightarrow[9, \ldots, 16]} \\
\end{array}$ & 5 & 130 & 312 & 4.88 \\
\hline \multirow{2}{*}{$\begin{array}{c}N_{t}=7 \\
N_{u}=3 \\
5 \mathrm{bpcu}\end{array}$} & Pro. & $\begin{array}{c}{[(1,2,6),(1,4,6),(1,2,5),(1,3,6),(1,2,7),(1,4,7),(1,2,4),(1,3,4)] \rightarrow[1, \ldots, 8]} \\
{[(1,5,7),(1,5,6),(2,5,6),(1,3,5),(2,5,7),(1,3,7),(2,3,5),(3,5,7)] \rightarrow[9, \ldots, 16]} \\
{[(2,4,6),(4,5,6),(2,4,5),(3,4,6),(2,4,7),(3,4,7),(2,3,4),(3,4,5)] \rightarrow[17, \ldots, 24]} \\
{[(2,6,7),(3,6,7),(2,3,6),(3,5,6),(2,3,7),(4,5,7),(5,6,7),(4,6,7)] \rightarrow[25, \ldots, 32]}\end{array}$ & 3 & 348 & 664 & 4.15 \\
\hline & Con. & $\begin{array}{r}{[(1,6,7),(3,6,7),(2,3,6),(1,4,7),(2,4,7),(1,3,4),(1,3,7),(1,2,6)] \rightarrow[1, \ldots, 8]} \\
{[(1,5,6),(2,4,6),(5,6,7),(2,5,7),(4,5,7),(3,4,5),(1,4,5),(3,5,6)] \rightarrow[9, \ldots, 16]} \\
{[(3,5,7),(4,5,6),(1,3,5),(2,4,5),(2,5,6),(2,3,5),(3,4,6),(1,5,7)] \rightarrow[17, \ldots, 24]} \\
{[(1,2,7),(1,3,6),(2,3,4),(3,4,7),(4,6,7),(2,3,7),(1,4,6),(2,6,7)] \rightarrow[25, \ldots, 32]}\end{array}$ & 3 & 354 & 1056 & 6.60 \\
\hline \multirow{2}{*}{$\begin{array}{c}N_{t}=8 \\
N_{u}=2 \\
4 \text { bpcu }\end{array}$} & Pro. & $\begin{array}{c}{[(1,5),(1,6),(1,7),(1,8),(2,5),(2,6),(2,7),(2,8)] \rightarrow[1, \ldots, 8]} \\
{[(3,5),(3,6),(3,7),(3,8),(4,5),(4,6),(4,7),(4,8)] \rightarrow[9, \ldots, 16]}\end{array}$ & 12 & 96 & 128 & 2 \\
\hline & Con. & $\begin{array}{l}{[(4,8),(6,8),(3,5),(3,7),(4,7),(5,6),(2,8),(3,4)] \rightarrow[1, \ldots, 8]} \\
{[(4,5),(3,8),(6,7),(4,6),(3,6),(5,7),(7,8),(5,8)] \rightarrow[9, \ldots, 16]}\end{array}$ & 12 & 130 & 312 & 4.88 \\
\hline \multirow[t]{2}{*}{$\begin{array}{c}N_{t}=8 \\
N_{u}=3 \\
5 \text { bpcu }\end{array}$} & Pro. & $\begin{array}{c}{[(1,2,7),(1,4,7),(1,3,4),(1,4,8),(1,2,6),(1,6,8),(1,3,6),(1,3,8)] \rightarrow[1, \ldots, 8]} \\
{[(1,5,7),(1,5,8),(3,4,5),(3,5,8),(1,2,5),(2,5,8),(3,4,6),(5,7,8)] \rightarrow[9, \ldots, 16]} \\
{[(2,4,7),(2,3,7),(2,3,4),(2,4,8),(2,6,7),(2,6,8),(3,6,7),(4,6,8)] \rightarrow[17, \ldots, 24]} \\
{[(2,3,5),(4,5,7),(4,5,6),(4,7,8),(2,5,6),(6,7,8),(5,6,7),(3,7,8)] \rightarrow[25, \ldots, 32]}\end{array}$ & 24 & 240 & 416 & 2.6 \\
\hline & Con. & $\begin{array}{c}{[(2,7,8),(4,7,8),(3,4,7),(2,5,8),(3,5,8),(2,4,5),(2,4,8),(2,3,7)] \rightarrow[1, \ldots, 8]} \\
{[(2,6,7),(3,5,7),(6,7,8),(3,6,8),(5,6,8),(4,5,6),(2,5,6),(4,6,7)] \rightarrow[8,9, \ldots, 15]} \\
{[(4,6,8),(5,6,7),(2,4,6),(3,5,6),(3,6,7),(3,4,6),(4,5,7),(2,6,8)] \rightarrow[16,17, \ldots, 23]} \\
{[(2,3,8),(2,4,7),(3,4,5),(4,5,8),(5,7,8),(3,4,8),(2,5,7),(3,7,8)] \rightarrow[24,25, \ldots, 31]}\end{array}$ & 24 & 354 & 1056 & 6.60 \\
\hline \multirow[t]{2}{*}{$\begin{array}{c}N_{t}=8 \\
N_{u}=4 \\
6 \text { bpcu }\end{array}$} & Pro. & $\begin{array}{c}{[(1,2,3,7),(1,2,5,7),(1,2,6,8),(1,2,6,7),(2,3,5,7),(2,3,6,7)] \rightarrow[1, \ldots, 6]} \\
{[(2,3,6,8),(2,3,7,8),(1,2,3,6),(1,2,4,7),(1,4,6,8),(1,2,4,6)] \rightarrow[7, \ldots, 12]} \\
{[(2,3,5,6),(2,3,4,6),(2,4,6,8),(2,4,6,7),(1,2,3,8),(1,2,5,8)] \rightarrow[13, \ldots, 18]} \\
{[(1,3,5,8),(1,2,4,8),(2,3,5,8),(2,3,4,7),(2,3,4,8),(2,4,5,8)] \rightarrow[19, \ldots, 24]} \\
{[(1,2,3,5),(1,2,4,5),(3,5,6,8),(2,4,7,8),(2,3,4,5),(2,4,5,7)] \rightarrow[25, \ldots, 30]} \\
{[(2,5,6,8)(2,4,5,6),(1,3,6,7),(1,5,6,7),(5,6,7,8),(1,6,7,8)] \rightarrow[31, \ldots, 36]} \\
{[(3,5,6,7),(2,5,6,7),(3,6,7,8),(2,6,7,8),(1,3,5,6),(1,4,6,7)] \rightarrow[37, \ldots, 42]} \\
{[(1,4,5,6),(4,6,7,8),(1,3,4,6),(3,4,6,7),(3,4,6,8),(4,5,6,7)] \rightarrow[43, \ldots, 48]} \\
{[(1,3,7,8),(1,5,7,8),(1,3,4,8),(1,4,7,8),(3,5,7,8),(2,5,7,8)] \rightarrow[49, \ldots, 54]} \\
{[(3,4,5,8),(3,4,7,8),(1,3,4,5),(1,3,4,7),(1,5,6,8),(1,4,5,7)] \rightarrow[55, \ldots, 60]} \\
{[(3,4,5,6),(3,4,5,7),(4,5,6,8),(4,5,7,8)] \rightarrow[61,62,63,64]}\end{array}$ & 6 & 928 & 1916 & 4.99 \\
\hline & Con. & $\begin{aligned} & {[(1,2,4,8),(1,2,6,7),(1,6,7,8),(2,4,7,8),(1,3,4,6),(4,6,7,8]) } \rightarrow[1, \ldots, 6] \\
& {[(1,2,5,6),(1,5,7,8),(3,4,6,8),(3,4,7,8),(2,3,4,5),(3,4,5,6)] } \rightarrow[7, \ldots, 12] \\
& {[(2,5,7,8),(4,5,6,7),(1,4,5,8),(1,3,6,8),(2,3,6,8),(1,4,5,7)] } \rightarrow[13, \ldots, 18] \\
& {[(1,3,4,8),(2,5,6,7),(2,3,4,7),(3,5,6,8),(1,3,5,7),(3,6,7,8)] } \rightarrow[19, \ldots, 24] \\
& {[(1,5,6,8),(1,2,4,6),(2,3,5,8),(1,2,7,8),(2,4,5,6),(1,3,4,5)] } \rightarrow[25, \ldots, 30] \\
& {[(1,4,6,7),(2,4,6,8),(2,4,6,7),(1,4,6,8),(1,4,5,6),(2,5,6,8)] } \rightarrow[31, \ldots, 36] \\
& {[(1,3,7,8),(1,2,5,8),(1,2,4,7),(3,4,5,8),(1,3,5,8),(2,3,5,6)] } \rightarrow[37, \ldots, 42] \\
& {[(2,3,6,7),(1,3,4,7),(3,5,6,7),(1,5,6,7),(2,3,4,8),(2,4,5,7)] } \rightarrow[43, \ldots, 48] \\
& {[(3,4,5,7),(2,4,5,8),(4,5,7,8),(1,3,5,6),(4,5,6,8),(2,3,5,7)] } \rightarrow[49, \ldots, 54] \\
& {[(3,4,6,7),(2,3,4,6),(1,2,5,7),(1,3,6,7),(5,6,7,8),(3,5,7,8)] } \rightarrow[55, \ldots, 60] \\
& {[(2,6,7,8),(1,4,7,8),(1,2,6,8),(2,3,7,8)] \rightarrow[61,62,63,64] }\end{aligned}$ & 6 & 952 & 3360 & 8.75 \\
\hline \multirow{2}{*}{$\begin{array}{c}N_{t}=10 \\
N_{u}=2 \\
5 \text { bpcu }\end{array}$} & Pro. & $\begin{array}{c}(1,5),(1,6),(1,8),(1,10),(4,5),(5,8),(4,8),(5,10)] \rightarrow[1, \ldots, 8] \\
{[(1,4),(1,7),(4,9),(7,10),(4,7),(6,10),(4,10),(8,10)] \rightarrow[9, \ldots, 16]} \\
{[(2,5),(2,6),(2,8),(2,3),(5,9),(3,6),(8,9),(3,8)] \rightarrow[17, \ldots, 24]} \\
{[(7,9),(2,7),(2,9),(3,7),(6,9),(6,7),(3,9),(3,10)] \rightarrow[25, \ldots, 32]}\end{array}$ & 13 & 348 & 656 & 4.1 \\
\hline & Con. & $\begin{array}{l}{[(2,9),(7,8),(7,10),(3,8),(6,10),(9,10),(5,9),(3,5)] \rightarrow[1, \ldots, 8]} \\
{[(4,10),(3,6),(6,8),(4,9),(3,7),(5,8),(2,8),(4,7)] \rightarrow[9, \ldots, 16]} \\
{[(5,7),(2,7),(3,10),(8,9),(4,8),(3,4),(4,6),(6,7)] \rightarrow[17, \ldots, 24]} \\
{[(3,9),(5,6),(4,5),(5,10),(8,10),(6,9),(7,9),(2,10)] \rightarrow[25, \ldots, 32]}\end{array}$ & 13 & 404 & 1154 & 7.21 \\
\hline
\end{tabular}

the conventional counterpart. $N_{r}=2, P=1, Q=2, M=2$ are employed for all the MS-GSTSK systems. For the case of $N_{u}=2$, it is observed from Fig. 9 that the proposed mapping principle based MS-GSTSK systems with $N_{t}=6,10,16$ outperforms the conventional counterparts by $1.2 \mathrm{~dB}, 1.2$ $\mathrm{dB}$ and $1.3 \mathrm{~dB}$ at $\mathrm{BER}=10^{-4}$, respectively. For the case of $N_{u}>2$, it is shown from Fig. 10 that the proposed scheme based MS-GSTSK systems with $\left(N_{t}, N_{u}\right)=(6,3),(12,3)$ and $(8,4)$ provides $1.8 \mathrm{~dB}, 2 \mathrm{~dB}$, and $1.5 \mathrm{~dB}$ gains over the corresponding conventional counterparts, respectively.

\section{B. Performance comparison of recent GIM schemes}

Figs. 11 and 12 compare the performances of the OFDM-IM system employing the proposed mapping principle to that of the conventional counterpart for $N_{t}=8$, where the channel model is the same as [30]. For the case of $M=1$, it is observed from Fig. 11 that the proposed mapping principle based OFDM-IM systems with $\left(N_{t}, N_{u}\right)=(8,2),(8,3),(8,4)$ outperforms the conventional counterparts by $2 \mathrm{~dB}, 3 \mathrm{~dB}$ and $2 \mathrm{~dB}$, respectively, while they still provides $0.8 \mathrm{~dB}, 1.8 \mathrm{~dB}$ and $1.3 \mathrm{~dB}$ gains for the case of $M=2$.

Finally, Fig. 13 compares the performances of the recent advanced orbital angular momentum (OAM) based GIM sys- 
TABLE II

BIT-TO-IC MAPPING FOR DIFFERENT GIM SCHEMES.

\begin{tabular}{|c|c|c|c|c|c|c|}
\hline \multicolumn{2}{|c|}{ Scheme } & $\begin{array}{c}\text { bit-to-IC mapping } \\
\text { IC } \text { set }: \mathbb{I} \rightarrow \text { bits index }\end{array}$ & $N_{\mathrm{re}}$ & $\delta(G)$ & $W\left(\mathbf{A}_{1}\right)$ & $\frac{W\left(\mathbf{A}_{1}\right)}{B 2^{B}}$ \\
\hline \multirow[t]{2}{*}{$\begin{array}{c}N_{t}=10 \\
N_{u}=3 \\
6 \text { bpcu }\end{array}$} & Pro. & $\begin{array}{c}(1,3,7),(1,4,7),(1,5,9),(1,2,7),(1,5,7),(2,3,7),(3,5,7),(2,3,6)] \rightarrow[1, \ldots, 8] \\
{[(7,9,10),(2,7,10),(5,6,9),(6,9,10),(5,7,10),(2,7,8),(5,6,7),(2,5,10)] \rightarrow[9, \ldots, 16]} \\
{[(1,3,10),(1,5,8),(1,6,8),(1,2,6),(3,5,8),(3,5,10),(1,5,6),(3,6,10)] \rightarrow[17, \ldots, 24]} \\
{[(5,8,9),(4,7,10),(1,6,10),(2,6,10),(5,8,10),(2,5,8),(5,6,10),(2,5,6)] \rightarrow[25, \ldots, 32]} \\
{[(1,3,9),(1,4,9),(1,9,10),(1,2,9),(3,7,9),(2,3,9),(4,5,7),(2,3,4)] \rightarrow[33, \ldots, 40]} \\
{[(7,8,9),(2,8,9),(6,7,9),(2,9,10),(7,8,10),(2,4,8),(6,7,8),(2,4,5)] \rightarrow[41, \ldots, 48]} \\
{[(1,3,8),(1,4,8),(1,8,10),(1,2,4),(3,8,9),(3,4,10),(3,6,8),(3,4,5)] \rightarrow[49, \ldots, 56]} \\
{[(4,6,9),(4,9,10),(6,8,9),(4,5,9),(4,6,7),(4,8,10),(4,6,8),(3,4,6)] \rightarrow[57, \ldots, 64]}\end{array}$ & 56 & 636 & 1262 & 3.29 \\
\hline & Con. & $\begin{array}{c}{[(2,9,10),(7,9,10),(6,7,9),(2,8,10),(6,8,10),(2,7,8),(4,7,10),(5,9,10)] \rightarrow[1, \ldots, 8]} \\
{[(3,4,8),(4,6,7),(3,5,7),(3,6,10),(2,7,10),(2,6,9),(7,8,9),(3,4,5)] \rightarrow[9, \ldots, 16]} \\
{[(4,6,10],(6,8,9),(4,7,8),(5,6,9),(5,7,8),(3,6,7),(3,9,10),(3,4,9)] \rightarrow[17, \ldots, 24]} \\
{[(4,5,6),(4,8,9),(5,8,10),(5,7,10),(3,8,10),(3,7,9),(3,6,8),(3,5,6)] \rightarrow[25, \ldots, 32]} \\
{[(3,6,9),(3,7,8),(3,7,10),(3,8,9),(5,8,9),(4,8,10),(5,6,7),(4,6,8)] \rightarrow[33, \ldots, 40]} \\
{[(5,7,9),(5,6,8),(4,5,8),(4,9,10),(5,6,10),(4,5,10),(4,6,9),(3,4,7)] \rightarrow[41, \ldots, 48]} \\
{[(3,4,6),(4,5,7),(2,6,10),(2,7,9),(4,7,9),(3,5,10),(4,5,9),(3,5,8)] \rightarrow[49, \ldots, 56]} \\
{[(3,5,9),(3,4,10),(6,7,8),(7,8,10),(8,9,10),(6,7,10),(2,8,9),(6,9,10)] \rightarrow[57, \ldots, 64]}\end{array}$ & 56 & 974 & 3376 & 8.79 \\
\hline \multirow[t]{2}{*}{$\begin{array}{c}N_{t}=12 \\
N_{u}=3 \\
7 \text { bpcu }\end{array}$} & Pro. & $\begin{array}{c}(1,2,8),(1,2,11),(1,2,7),(1,4,6),(1,2,10),(1,6,10),(1,2,6),(1,6,11)] \rightarrow[1, \ldots, 8] \\
{[(1,5,10),(1,4,8),(1,8,12),(1,8,11),(1,8,10),(1,4,10),(1,10,12),(1,6,12)] \rightarrow[9, \ldots, 16]} \\
{[(1,3,7),(1,3,11),(1,5,7),(1,5,11),(1,5,9),(1,3,9),(1,7,9),(1,9,1)] \rightarrow[17, \ldots, 24]} \\
{[(1,3,8),(1,3,4),(1,7,12),(1,4,7),(3,8,12),(3,9,11),(1,5,12),(1,9,1)] \rightarrow[25, \ldots, 32]} \\
{[((2,5,10),(5,6), 10),(2,6,9),(5,11),(2,6,1),(2,6,11),(2,6,12),(2,5,6)] \rightarrow[33, \ldots, 40]} \\
{[(3,5,10),(4,5,8),(2,4,5),(4,5,6),(5,8,10),(2,11,12),(4,6,12),(6,11,12)] \rightarrow[41, \ldots, 48]} \\
{[(5,7,10),(3,5,11),(2,5,9),(5,6,9),(3,5,12),(3,5,9),(2,5,12),(5,9,11)] \rightarrow[49, \ldots, 56]} \\
{[(3,5,8),(3,4,5),(4,5,7),(5,11,12),(5,8,12),(5,8,9),(4,5,12),(9,11,12)] \rightarrow[57, \ldots, 64]} \\
{[(2,8,9),(2,8,11),(2,7,9),(6,8,9),(2,3,10),(6,9,10),(3,6,12),(3,6,10)] \rightarrow[65, \ldots, 72]} \\
{[(4,8,9),(2,4,8),(6,7,8),(4,6,8),(4,9,10),(3,4,10),(6,8,12),(6,8,10)] \rightarrow[73, \ldots, 80]} \\
{[(2,3,9),(3,10,11),(3,6,7),(3,6,9),(7,9,10),(9,10,11),(3,10,12),(7,9,12)] \rightarrow[81, \ldots, 88]} \\
{[(2,3,8),(3,4,9),(4,7,9),(3,6,8),(8,9,10),(8,10,11),(7,8,12),(8,9,11)] \rightarrow[89, \ldots, 96]} \\
{[(2,7,8),(2,7,11),(6,7,9),(5,6,7),(2,7,10),(9,10,12),(7,10,12),(4,6,11)] \rightarrow[97, \ldots, 104]} \\
{[(4,7,10),(4,8,11),(2,4,9),(4,6,7),(2,4,10),(2,4,11),(2,4,12),(6,7,11)] \rightarrow[105, \ldots, 112]} \\
{[(2,3,7),(3,7,11),(3,7,8),(5,7,11),(2,3,12),(7,10,11),(3,7,12),(10,11,12)] \rightarrow[113, \ldots, 120]} \\
{[(5,7,8),(3,4,11),(4,7,12),(7,8,11),(8,10,12),(4,10,11),(4,9,12),(4,11,12)] \rightarrow[121, \ldots, 128]}\end{array}$ & 92 & 1860 & 4236 & 4.73 \\
\hline & Con. & $\begin{array}{c}{[(2,11,12),(9,11,12),(2,10,12),(8,10,11),(8,9,11),(8,10,12),(2,9,10),(9,10,12)] \rightarrow[1, \ldots, 8]} \\
{[(3,8,11),(4,8,9),(3,5,6),(3,4,10),(3,9,12),(2,8,12),(3,10,11),(2,9,11)] \rightarrow[9, \ldots, 16]} \\
{[(4,6,7),(4,10,11),(5,7,11),(3,4,12),(3,6,12),(4,5,12),(6,8,9),(5,6,10)] \rightarrow[17, \ldots, 24]} \\
{[(4,6,10),(7,11,12),(6,7,9),(4,5,8),(3,4,7),(5,8,12),(7,10,11),(5,9,11)] \rightarrow[25, \ldots, 32]} \\
{[(5,11,12),(5,10,11),(6,8,12),(4,6,11),(7,8,11),(3,7,9),(6,9,10),(5,6,12)] \rightarrow[33, \ldots, 40]} \\
{[(4,5,9),(4,7,10),(3,7,12),(7,8,9),(4,5,7),(5,6,7),(3,6,11),(3,5,8)] \rightarrow[41, \ldots, 48]} \\
{[(6,9,11),(3,6,8),(3,8,12),(4,5,6),(5,8,9),(3,5,10),(4,8,10),(7,8,10)] \rightarrow[49, \ldots, 56]} \\
{[(3,9,10),(6,10,12),(4,11,12),(3,5,7),(7,9,12),(4,8,11),(3,4,9),(4,9,12)] \rightarrow[57, \ldots, 64]} \\
{[(4,8,12),(4,9,10),(5,8,11),(6,10,11),(3,4,5),(3,11,12),(4,9,11),(3,8,9)] \rightarrow[65, \ldots, 72]} \\
{[(6,8,10),(6,9,12),(5,7,10),(3,6,9),(6,7,11),(4,5,11),(4,6,8),(5,9,10)] \rightarrow[73, \ldots, 80]} \\
{[(3,5,9),(3,7,11),(4,7,9),(7,8,12),(3,6,10),(7,9,10),(3,6,7),(4,7,12)] \rightarrow[81, \ldots, 88]} \\
{[(5,7,8),(5,6,9),(5,10,12),(3,5,11),(3,7,8),(6,8,11),(3,8,10),(6,11,12)] \rightarrow[89, \ldots, 96]} \\
{[(7,10,12),(3,4,8),(5,9,12),(3,7,10),(4,6,12),(5,6,8),(4,7,8),(4,7,11)] \rightarrow[97, \ldots, 104]} \\
{[(5,8,10),(6,7,8),(7,9,11),(3,4,6),(4,10,12),(5,7,12),(3,5,12),(6,7,10)] \rightarrow[105, \ldots, 112]} \\
{[(2,9,12),(3,10,12),(2,8,11),(3,9,11),(3,4,11),(4,6,9),(4,5,10),(5,7,9)] \rightarrow[113, \ldots, 120]} \\
{[(9,10,11),(8,9,10),(8,9,12),(6,7,12),(5,6,11),(2,10,11),(10,11,12),(8,11,12) \rightarrow[121, \ldots, 128]}\end{array}$ & 92 & 2686 & 10514 & 11.73 \\
\hline \multirow[t]{2}{*}{$\begin{array}{c}N_{t}=16 \\
N_{u}=2 \\
6 \text { bpcu }\end{array}$} & Pro. & $\begin{array}{l}{[(1,9),(1,10),(1,11),(1,12),(1,13),(1,14),(1,15),(1,16)] \rightarrow[1, \ldots, 8]} \\
{[(2,9),(2,10),(2,11),(2,12),(2,13),(2,14),(2,15),(2,16)] \rightarrow[9, \ldots, 16]} \\
{[(3,9),(3,10),(3,11),(3,12),(3,13),(3,14),(3,15),(3,16)] \rightarrow[17, \ldots, 24]} \\
{[(4,9),(4,10),(4,11),(4,12),(4,13),(4,14),(4,15),(4,16)] \rightarrow[25, \ldots, 32]} \\
{[(5,9),(5,10),(5,11),(5,12),(5,13),(5,14),(5,15),(5,16)] \rightarrow[33, \ldots, 40]} \\
{[(6,9),(6,10),(6,11),(6,12),(6,13),(6,14),(6,15),(6,16)] \rightarrow[41, \ldots, 48]} \\
{[(7,9),(7,10),(7,11),(7,12),(7,13),(7,14),(7,15),(7,16)] \rightarrow[49, \ldots, 56]} \\
{[(8,9),(8,10),(8,11),(8,12),(8,13),(8,14),(8,15),(8,16)] \rightarrow[57, \ldots, 64]}\end{array}$ & 56 & 896 & 1536 & 4 \\
\hline & Con. & $\begin{array}{c}{[(5,10),(5,12),(5,14),(5,16),(8,9),(7,8),(9,16),(10,15)] \rightarrow[1, \ldots, 8]} \\
{[(8,11),(8,13),(8,15),(9,14),(6,9),(11,12),(7,10),(11,16)] \rightarrow[9, \ldots, 16]} \\
{[(10,13),(6,11),(12,15),(5,8),(10,11),(11,14),(13,16),(7,13)] \rightarrow[17, \ldots, 24]} \\
{[(9,12),(12,13),(14,15),(9,10),(7,16),(6,15),(7,14),(6,12)] \rightarrow[25, \ldots, 32]} \\
{[(7,12),(15,16),(11,13),(9,11),(6,14),(13,15),(12,14),(10,12)] \rightarrow[33, \ldots, 40]} \\
{[(12,16),(7,15),(6,13),(6,7),(5,9),(13,14),(6,10),(8,10)] \rightarrow[41, \ldots, 48]} \\
{[(7,11),(14,16),(6,16),(6,8),(9,15),(8,14),(8,12),(9,13)] \rightarrow[49, \ldots, 56]} \\
{[(10,14),(10,16),(11,15),(7,9),(8,16),(5,15),(5,13),(5,11)] \rightarrow[57, \ldots, 64]}\end{array}$ & 56 & 1242 & 4072 & 10.6 \\
\hline
\end{tabular}

tem [3] employing the proposed mapping principle to that of the conventional counterpart. $N_{t}=6, N_{u}=2, N_{r}=6$ and $N_{t}=8, N_{u}=2, N_{r}=8$ are used for the OAM-GIM system, and the channel model is the same as [3]. It is shown from Fig. 13 that the performance of the proposed mapping principle based OAM-GIM still outperforms the conventional counterpart for both the cases of $M=1$ and $M=2$.

In a word, the performance gains of the proposed mapping method over the conventional counterpart under different configurations are summarized in Table V.

\section{CONCLUSIONS}

In this paper, we investigated the graph theory assisted bit-to-IC gray coding for GIM systems, where both the IC selection and bit-to-IC mapping are taken into consideration to improve the ABEP by minimizing the average HD. Specifically, we transformed the IC selection problem into searching a subgraph with the minimum degree and designed a low-complexity MDF algorithm with the complexity order of $O(N)$. For the selected IC, we reformulated the bit-to- 


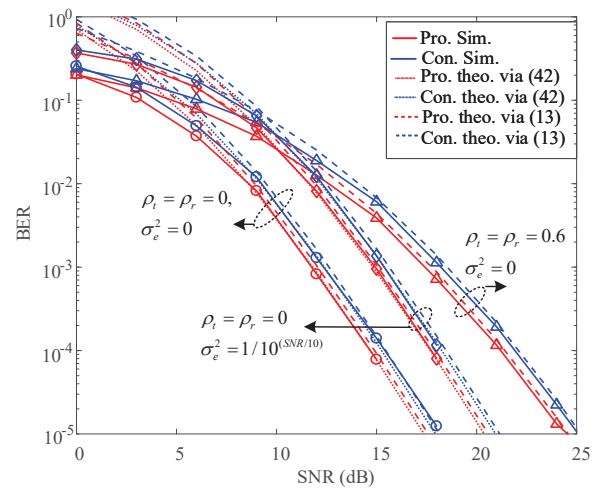

Fig. 4. Performance comparison of the proposed and conventional mapping principles for GSM systems having $N_{t}=6, N_{u}=2, N_{r}=6, M=2$ over different Rayleigh fading channels.

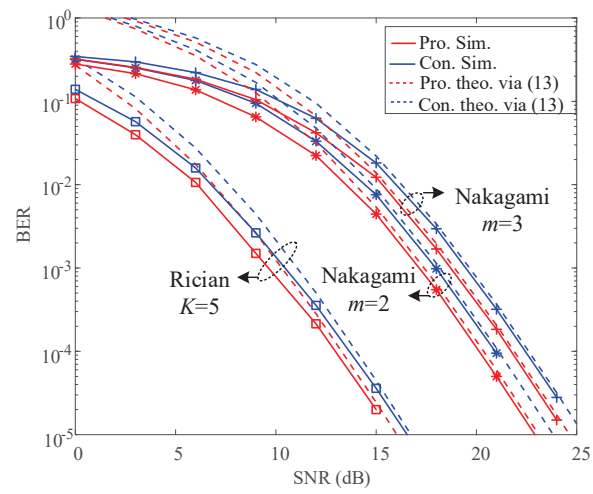

Fig. 5. Performance comparison of the proposed and conventional mapping principles for GSM systems having $N_{t}=6, N_{u}=2, N_{r}=6, M=2$ over Rician and Nakagami-m channels.

IC mapping problem into searching a minimum weighted subgraph from a weighted completed graph and designed a low-complexity MWF algorithm with the complexity order of $O\left(N^{2}\right)$. It is worth noting that, the proposed IC-selection and bit-to-IC mapping algorithms are straightforward for any GIM mapping. Both simulation and theoretical results show that the GIM systems employing our proposed scheme provide significant performance gains over the conventional counterpart without bringing extra complexity.

\section{APPENDIX A}

PEP EXPRESSIONS FOR DIFFERENT CHANNEL MODELS

\section{A. PEP of Rayleigh channel}

In the Rayleigh channel, the elements of $\mathbf{H}$ are independent and follow the Gaussian distribution as $\mathcal{C N}(0,1)$, and we have

$$
\overline{\mathbf{u}}=\mathbf{0}_{N_{r} N_{t} \times 1}, \mathbf{R}_{\mathbf{u}}=\mathbf{I}_{N_{r} N_{t}} .
$$

According to (11) and (13), the accurate and approximate PEPs of Rayleigh channel can be expressed as

$$
\begin{aligned}
& P\left(\mathbf{X}^{i} \rightarrow \mathbf{X}^{j}\right)=\frac{1}{\pi} \int_{0}^{\frac{\pi}{2}}\left|\mathbf{I}+\frac{\mathbf{B}}{4\left(\sigma^{2}+\left\|\mathbf{X}^{i}\right\|^{2} \sigma_{e}^{2}\right) \sin ^{2} \theta}\right|^{-1} d_{\theta} \\
& =\frac{1}{\pi} \int_{0}^{\frac{\pi}{2}} \prod_{k=1}^{\kappa}\left(1+\frac{\lambda_{k}}{4\left(\sigma^{2}+\left\|\mathbf{X}^{i}\right\|^{2} \sigma_{e}^{2}\right) \sin ^{2} \theta}\right)^{-N_{r}} d_{\theta},
\end{aligned}
$$
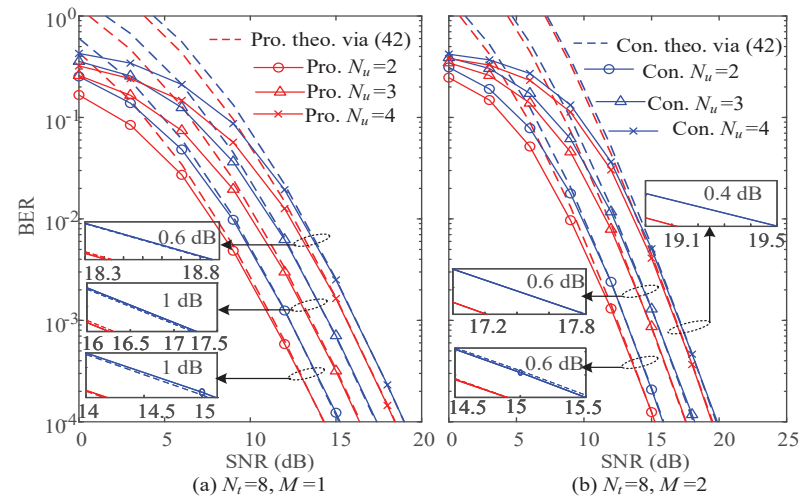

Fig. 6. Performance comparison of the proposed and conventional mapping principles for GSM systems having $N_{t}=8$.
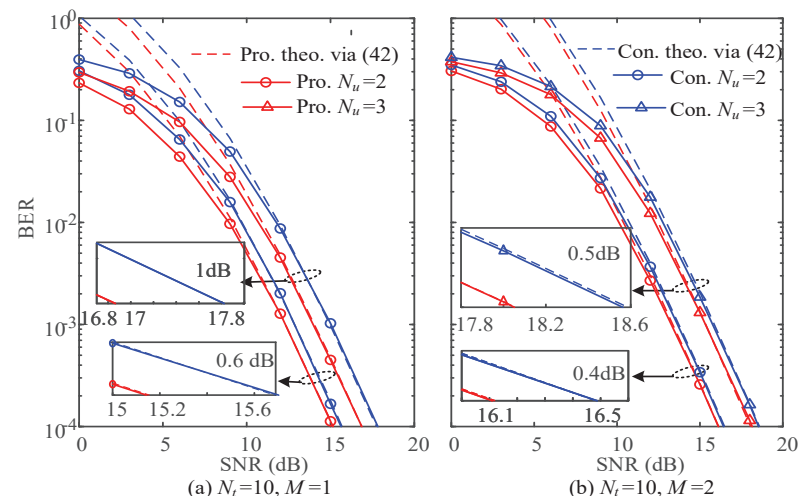

Fig. 7. Performance comparison of the proposed and conventional mapping principles for GSM systems having $N_{t}=10$.

and

$$
\begin{aligned}
& P\left(\mathbf{X}^{i} \rightarrow \mathbf{X}^{j}\right) \approx \frac{1}{12} \prod_{k=1}^{\kappa}\left(1+\frac{\lambda_{k}}{4\left(\sigma^{2}+\left\|\mathbf{X}^{i}\right\|^{2} \sigma_{e}^{2}\right)}\right)^{-N_{r}} \\
& +\frac{1}{4} \prod_{k=1}^{\kappa}\left(1+\frac{\lambda_{k}}{3\left(\sigma^{2}+\left\|\mathbf{X}^{i}\right\|^{2} \sigma_{e}^{2}\right)}\right)^{-N_{r}},
\end{aligned}
$$

where $\lambda_{k}$ is the $k$-th eigenvalue of $\triangle \triangle^{H}$ with $\Delta=\mathbf{X}^{i}-\mathbf{X}^{j}$. Furthermore, Eq. (40) can be further simplified for GSM by [24]

$$
P\left(\mathbf{X}^{i} \rightarrow \mathbf{X}^{j}\right)_{\mathrm{GSM}}=\gamma(\bar{\varsigma})^{N_{r}} \sum_{k=0}^{N_{r}-1}\left(\begin{array}{c}
N_{r}-1+k \\
k
\end{array}\right)[1-\gamma(\bar{\varsigma})]^{k},
$$

with $\gamma(\bar{\zeta})=\frac{1}{2}\left(1-\sqrt{\frac{\bar{\zeta} / 2}{1+\bar{\zeta} / 2}}\right)$ and $\bar{\varsigma}=\frac{\left\|\mathbf{X}^{i}-\mathbf{X}^{j}\right\|^{2}}{2\left(\sigma^{2}+\left\|\mathbf{X}^{i}\right\|^{2} \sigma_{e}^{2}\right)}$.

\section{B. PEP of Nakagami-m channel}

In the Nakagami- $m$ channel, the element of $r$-th row and $t$-th column of $\mathbf{H}$ can be expressed as [46]

$$
h_{r t}=\sqrt{\sum_{i=1}^{m}\left|x_{i}\right|^{2}}+j \sqrt{\sum_{i=1}^{m}\left|y_{i}\right|^{2}},
$$




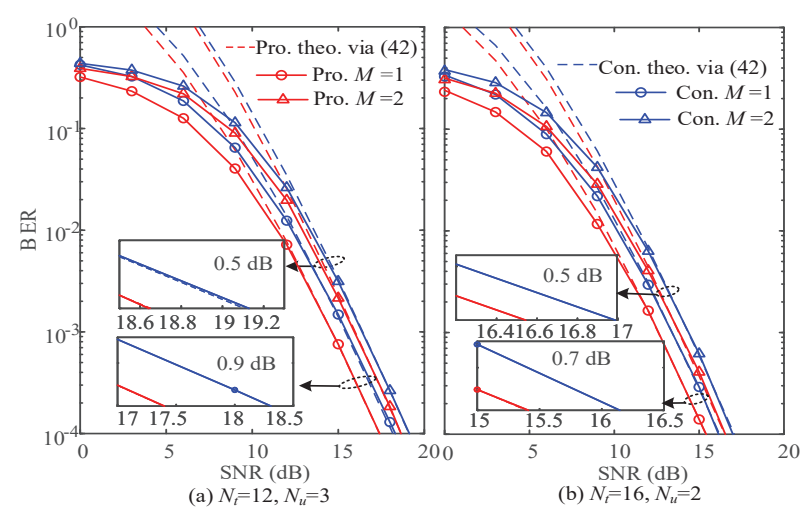

Fig. 8. Performance comparison of the proposed and conventional mapping principles for GSM systems having $N_{t}=12$ and $N_{t}=16$.

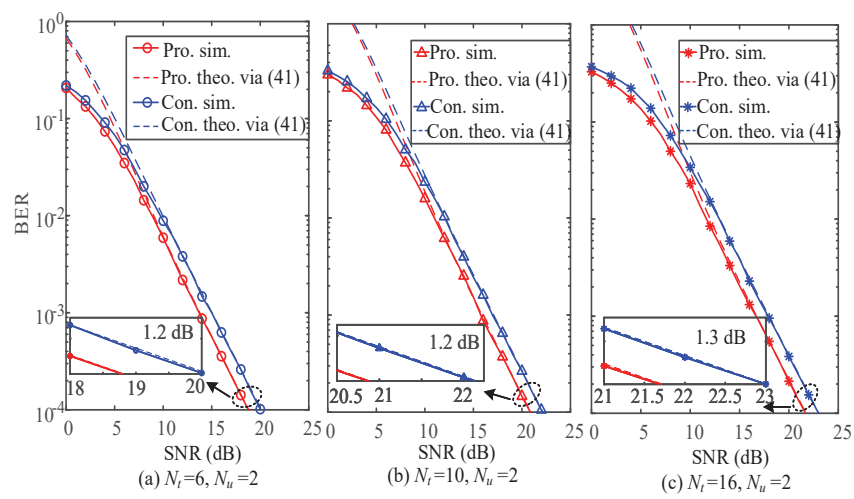

Fig. 9. Performance comparison of the proposed and conventional mapping principles for MS-GSTSK systems having $P=1, Q=2, M=2$.

where $x_{i}, y_{i} \in \mathcal{C N}(0,1 / 2 m)$. Its mean value and variance can be obtained by [48]

Hence, we have

$$
\begin{aligned}
& \mathbf{E}\left(h_{r t}\right)=\left(\frac{\Gamma(m / 2+1 / 2)}{\Gamma(m / 2) \sqrt{m / 2}} \exp \left(j \frac{\pi}{4}\right)\right) \\
& \operatorname{Var}\left(h_{r t}\right)=\mathbf{E}\left(\left|h_{r t}\right|^{2}\right)-\left|\mathbf{E}\left(h_{r t}\right)\right|^{2} \\
& =\frac{1}{2 m} 2 m-\left(\frac{\Gamma(m / 2+1 / 2)}{\Gamma(m / 2) \sqrt{m / 2}}\right)^{2} \\
& =1-\left(\frac{\Gamma(m / 2+1 / 2)}{\Gamma(m / 2) \sqrt{m / 2}}\right)^{2} .
\end{aligned}
$$

$$
\begin{aligned}
& \overline{\mathbf{u}}=\left(\frac{\Gamma(m / 2+1 / 2)}{\Gamma(m / 2) \sqrt{m / 2}} \exp \left(j \frac{\pi}{4}\right)\right) \mathbf{1}_{N_{r} N_{t} \times 1}, \\
& \mathbf{R}_{\mathbf{u}}=\left(1-\left(\frac{\Gamma(m / 2+1 / 2)}{\Gamma(m / 2) \sqrt{m / 2}}\right)^{2}\right) \mathbf{I}_{N_{r} N_{t}} .
\end{aligned}
$$

The PEP of Nakagami- $m$ channel can be obtained by (13) via (45).

\section{PEP of Rician fading channel}

According to [46], the Rician fading channel matrix can be expressed by [46]

$$
\mathbf{H}=\sqrt{\frac{K}{K+1}} \overline{\mathbf{H}}+\sqrt{\frac{1}{K+1}} \tilde{\mathbf{H}},
$$

where $\overline{\mathbf{H}}$ is a fixed matrix with all elements being one, $\tilde{\mathbf{H}}$ is a varied matrix whose elements obey the complex Gaussian
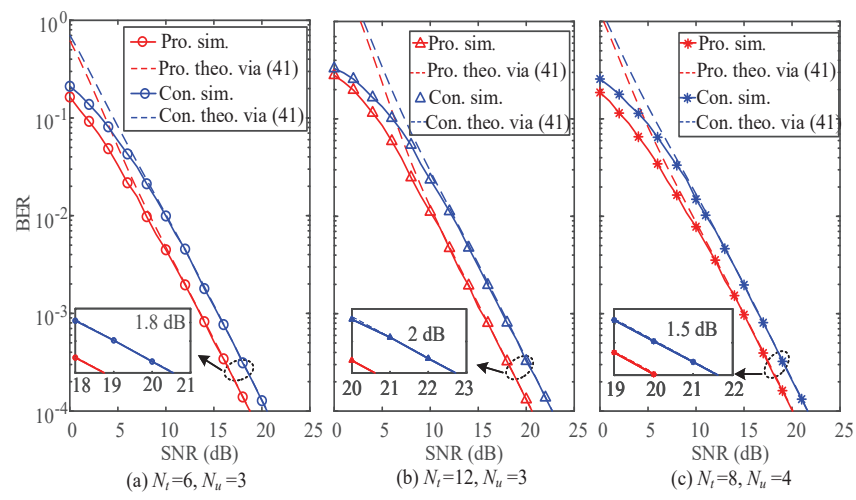

Fig. 10. Performance comparison of the proposed and conventional mapping principles for MS-GSTSK systems having $P=1, Q=2, M=2$.
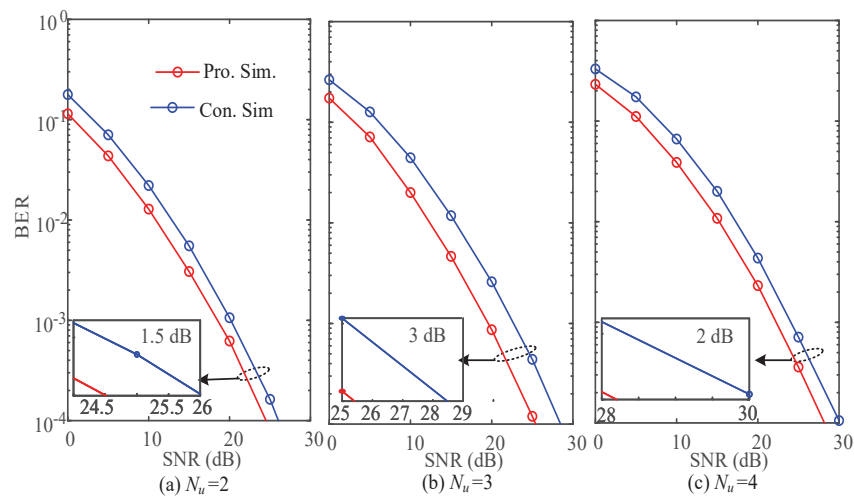

Fig. 11. Performance comparison of the proposed and conventional mapping principles for OFDM-IM systems having $N_{t}=8, M=1$.

distributions associated with $\mathcal{C N}(0,1)$. Hence the element of $r$-th row and $t$-th column of $\mathbf{H}$ is expressed as

$$
h_{r t}=\sqrt{\frac{K}{K+1}}+\sqrt{\frac{1}{K+1}} \tilde{h}_{r t},
$$

where $\tilde{h}_{r t} \in \mathcal{C N}(0,1)$ is the $r$-th row and $t$-th column of $\tilde{\mathbf{H}}$. The mean and variance of $h_{r t}$ are expressed as

$$
\begin{aligned}
& \mathbf{E}\left(h_{r t}\right)=\sqrt{\frac{K}{K+1}} \\
& \operatorname{Var}\left(h_{r t}\right)=\mathbf{E}\left(\left|h_{r t}\right|^{2}\right)-\left|\mathbf{E}\left(h_{r t}\right)\right|^{2} \\
& =\mathbf{E}\left[\left(\sqrt{\frac{K}{K+1}}+\sqrt{\frac{1}{K+1}} \tilde{h}_{r t}\right)\left(\sqrt{\frac{K}{K+1}}+\sqrt{\frac{1}{K+1}} \tilde{h}_{r t}\right)^{H}\right] \\
& -\left|\mathbf{E}\left(h_{r t}\right)\right|^{2} \\
& =\frac{K}{K+1}+\frac{1}{K+1} \mathbf{E}\left(\left|\tilde{h}_{r t}\right|^{2}\right)-\frac{K}{K+1} \\
& =\frac{1}{K+1} .
\end{aligned}
$$

Hence the value of $\overline{\mathbf{u}}$ and $\mathbf{R}_{\mathbf{u}}$ can be obtained by

$$
\begin{aligned}
& \overline{\mathbf{u}}=\sqrt{\frac{K}{K+1}} \times \mathbf{1}_{N_{r} N_{t} \times 1}, \\
& \mathbf{R}_{\mathbf{u}}=\sqrt{\frac{1}{K+1}} \times \mathbf{I}_{N_{r} N_{t}} .
\end{aligned}
$$

The PEP of Rician fading channel can be obtained by (13) via (49). 


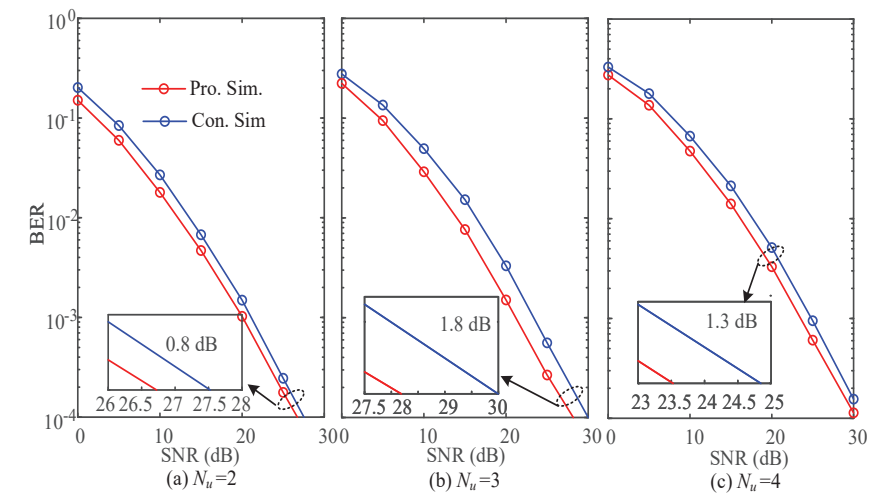

Fig. 12. Performance comparison of the proposed and conventional mapping principles for OFDM-IM systems having $N_{t}=8, M=2$.
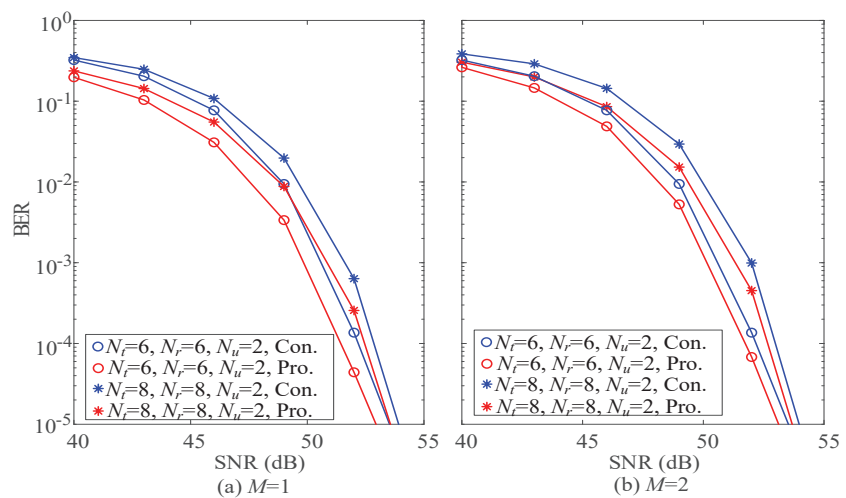

Fig. 13. Performance comparison of the proposed and conventional mapping principles for GIM system of [3] having $M=2$.

\section{PEP of correlated channel}

According to [46], the correlated channel can be expressed as

$$
\mathbf{H}=\mathbf{H}_{R} \tilde{\mathbf{H}} \mathbf{H}_{T},
$$

where $\mathbf{H}_{R} \in \mathbb{C}^{N_{r} \times N_{r}}$ and $\mathbf{H}_{t} \in \mathbb{C}^{N_{t} \times N_{t}}$ can be obtained by

$$
h_{R}^{p, q}=\rho_{r}{ }^{|p-q|}, p, q \in\left(1, \ldots, N_{r}\right) \text {, }
$$

and

$$
h_{T}^{u, v}=\rho_{t}^{|u-v|}, u, v \in\left(1, \ldots, N_{t}\right) .
$$

Hence, we have $\mathbf{u}=\operatorname{vec}\left(\mathbf{H}^{H}\right)=\left[\left(\mathbf{H}_{R}^{H}\right)^{T} \otimes \mathbf{H}_{T}^{H}\right] \operatorname{vec}\left(\tilde{\mathbf{H}}^{H}\right)$. The value of $\overline{\mathbf{u}}$ and $\mathbf{R}_{\mathbf{u}}$ can be obtained by

$$
\begin{aligned}
& \overline{\mathbf{u}}=\mathbf{E}\left(\left[\left(\mathbf{H}_{R}^{H}\right)^{T} \otimes \mathbf{H}_{T}^{H}\right] \operatorname{vec}\left(\tilde{\mathbf{H}}^{H}\right)\right) \\
& =\left[\left(\mathbf{H}_{R}^{H}\right)^{T} \otimes \mathbf{H}_{T}^{H}\right] \mathbf{E}\left(\operatorname{vec}\left(\tilde{\mathbf{H}}^{H}\right)\right)=\mathbf{O}_{N_{r} N_{t} \times 1},
\end{aligned}
$$

and

$$
\begin{aligned}
& \mathbf{R}_{\mathbf{u}}=\mathbf{E}\left(\mathbf{u u}^{H}\right) \\
& =\mathbf{E}\left(\left[\left(\mathbf{H}_{R}^{H}\right)^{T} \otimes \mathbf{H}_{T}^{H}\right]\left[\left(\mathbf{H}_{R}^{H}\right)^{T} \otimes \mathbf{H}_{T}^{H}\right]^{H}\right) \\
& =\left[\left(\mathbf{H}_{R}^{H}\right)^{T} \otimes \mathbf{H}_{T}^{H}\right] \mathbf{E}\left(\operatorname{vec}\left(\tilde{\mathbf{H}}^{H}\right) \operatorname{vec}\left(\tilde{\mathbf{H}}^{H}\right)\right)\left[\left(\mathbf{H}_{R}^{H}\right)^{T} \otimes \mathbf{H}_{T}^{H}\right]^{H} \\
& =\left[\left(\mathbf{H}_{R}^{H}\right)^{T} \otimes \mathbf{H}_{T}^{H}\right] \mathbf{I}_{N_{r} N_{t}}\left[\left(\mathbf{H}_{R}^{H}\right)^{T} \otimes \mathbf{H}_{T}^{H}\right]^{H} \\
& =\left[\left(\mathbf{H}_{R}^{H}\right)^{T} \otimes \mathbf{H}_{T}^{H}\right]\left[\left(\mathbf{H}_{R}^{H}\right)^{T} \otimes \mathbf{H}_{T}^{H}\right]^{H} \text {. }
\end{aligned}
$$

TABLE V

PERFORMANCE ADVANTAGE OVER THE CONVENTIONAL MAPPING METHOD

\begin{tabular}{|c|c|c|c|c|}
\hline \multicolumn{2}{|c|}{ Setup } & GSM & MS-GSTSK & OFDM-IM \\
\hline $\begin{array}{c}N_{t}=8 \\
N_{u}=2\end{array}$ & $M=1$ & $1 \mathrm{~dB}$ & - & $1.5 \mathrm{~dB}$ \\
\cline { 2 - 5 } & $M=2$ & $0.6 \mathrm{~dB}$ & - & $0.8 \mathrm{~dB}$ \\
\hline $\begin{array}{c}N_{t}=8 \\
N_{u}=3\end{array}$ & $M=1$ & $1 \mathrm{~dB}$ & - & $3 \mathrm{~dB}$ \\
\cline { 2 - 5 } & $M=2$ & $0.6 \mathrm{~dB}$ & - & $1.8 \mathrm{~dB}$ \\
\hline $\begin{array}{c}N_{t}=8 \\
N_{u}=4\end{array}$ & $M=1$ & $0.6 \mathrm{~dB}$ & - & $2 \mathrm{~dB}$ \\
\cline { 2 - 5 } & $M=2$ & $0.4 \mathrm{~dB}$ & $1.5 \mathrm{~dB}$ & $1.3 \mathrm{~dB}$ \\
\hline $\begin{array}{c}N_{t}=10 \\
N_{u}=2\end{array}$ & $M=2$ & $0.4 \mathrm{~dB}$ & $1.2 \mathrm{~dB}$ & - \\
\hline $\begin{array}{c}N_{t}=12 \\
N_{u}=3\end{array}$ & $M=2$ & $0.5 \mathrm{~dB}$ & $2 \mathrm{~dB}$ & - \\
\hline $\begin{array}{c}N_{t}=16 \\
N_{u}=2\end{array}$ & $M=2$ & $0.5 \mathrm{~dB}$ & $1.3 \mathrm{~dB}$ & - \\
\hline
\end{tabular}

The accurate and approximate PEPs of correlated channel can be obtained by (11) and (13) as

$$
P\left(\mathbf{X}^{i} \rightarrow \mathbf{X}^{j}\right)=\frac{1}{\pi} \int_{0}^{\frac{\pi}{2}}\left|\mathbf{I}+\frac{\mathbf{R}_{\mathbf{u}} \mathbf{B}}{4\left(\sigma^{2}+\sigma_{e}^{2}\right) \sin ^{2} \theta}\right|^{-1} d_{\theta},
$$

and

$$
P\left(\mathbf{X}^{i} \rightarrow \mathbf{X}^{j}\right) \approx \frac{1}{12}\left|\mathbf{I}+\frac{\mathbf{R}_{\mathbf{u}} \mathbf{B}}{4\left(\sigma^{2}+\sigma_{e}^{2}\right)}\right|^{-1}+\frac{1}{4}\left|\mathbf{I}+\frac{\mathbf{R}_{\mathbf{u}} \mathbf{B}}{3\left(\sigma^{2}+\sigma_{e}^{2}\right)}\right|_{(56)}^{-1}
$$

\section{REFERENCES}

[1] T. Mao, Q. Wang, Z. Wang and S. Chen, "Novel index modulation techniques: A survey," IEEE Commun. Surveys Tuts., vol. 21, no. 1, pp. 315-348, First Quart. 2019.

[2] E. Basar, "Index modulation techniques for $5 \mathrm{G}$ wireless networks," IEEE Commun. Magazine, vol. 54, no. 7, pp. 168-175, July 2016.

[3] E. Basar, "Orbital angular momentum with index modulation," IEEE Trans. Wireless Commun., vol. 17, no. 3, pp. 2029-2037, Mar. 2018.

[4] M. C. Gursoy, E. Basar, A. E. Pusane, and T. Tugcu, "Index modulation for molecular communicationnvia diffysion systems," IEEE Trans. Commun., vol. 67, no. 5, pp. 3337-3350, May 2019.

[5] Q. Li, M. Wen, S. Dang, E. Basar, H. V. Poor and F. Chen, "Opportunistic spectrum sharing based on OFDM with index modulation," IEEE Trans. Wireless Commun., vol. 19, no. 1, pp. 192-204, Jan. 2020.

[6] M. Au, G. Kaddoum, M. Alam, E. Basar and F. Gagnon,"Joint codefrequency index modulation for IoT and multi-user communications," IEEE J. of Sel. Topics in Signal Process., vol. 13, no. 6, pp. 1223-1236, Oct. 2019.

[7] F. J. Escribano, A. Wagemakers, G. Kaddoum and J. Evangelista, "A spatial time-frequency hopping index modulated scheme in turbulencefree optical wireless communication channels," IEEE Trans. Commun., 10.1109/TCOMM.2020.2987561, Apr. 2020.

[8] F. J. Escribano, A. Wagemakers, G. Kaddoum and J. Evangelista, "Design and performance analysis of an index time-frequency modulation scheme for optical communications," IEEE J. of Sel. Topics in Signal Process., vol. 13, no. 6, pp. 1403-1416, Oct. 2019.

[9] E. Basar, "Recondigurable intelligent surface-based index modulation: A new beyond MIMO paradigm for 6G," IEEE Trans. Commun., 2020, Doi: 10.1109/TCOMM.2020.2971486. 
[10] R. Mesleh, H. Haas, S. Sinanovic, C. W. Ahn, and S. Yun, "Spatial modulation," IEEE Trans. Veh. Technol., vol. 57, no. 4, pp. 2228-2241, July 2008

[11] M. Di Renzo, H. Haas, and P. M. Grant, "Spatial modulation for multiple-antenna wireless systems: A survey," IEEE Commun. Mag., vol. 49, no. 12, pp. 182-191, Dec. 2011.

[12] M. Di Renzo, H. Haas, A. Ghrayeb, S. Sugiura, and L. Hanzo, "Spatial modulation for generalized MIMO: challenges, opportunities and implementation," Proceedings of the IEEE, vol. 102, no. 1, pp. 56103, Jan. 2014

[13] P. Yang, M. Di Renzo, Y. Xiao, S. Q. Li and L. Hanzo, "Design guidelines for spatial modulation," IEEE Commun. Surveys Tuts., vol. 17, no. 1, pp. 6-26, First Quart. 2015.

[14] P. Yang, Y. Xiao, Y. L. Guan, K. V. S. Hari, A. Chockalingam, S. Sugiura, H. Haas, M. Di Renzo, C. Masouros, Z. Liu, L. Xiao, S. Li, and L. Hanzo, "Single-carrier spatial modulation: A promising design for large-scale broadband antenna systems," IEEE Commun. Surveys Tuts., vol. 18, no. 3, pp. 1687-1716, Feb. 2016.

[15] J. Wang, S. Jia, and J. Song, "Generalised spatial modulation with multiple active transmit antennas and low complexity detection scheme," IEEE Trans. Wireless Commun., vol. 11, no. 4, pp. 1605-1615, Apr 2012.

[16] W. O. Popoola, E. Poves and H. Haas, "Error performance of generalised space shift keying for indoor visible light communications," IEEE Trans. Commun., vo. 61, no. 5, pp. 1968-1976, May 2013.

[17] N. Ishikawa, R. Rajashekar, S. Sugiura and L. Hanzo, "Generalized spatial modulation based reduced-RF-chain millimeter-wave communications," IEEE Trans. Veh. Technol., vol. 66, no. 1, pp. 879-883, Jan. 2017.

[18] T. L. Narasimhan, P. Raviteja and A. Chockalingam, "Generalized spatial modulation in large-scale multiuser MIMO systems," IEEE Trans. Wireless Commun. vol. 14, no. 7, pp. 1536-1276, Mar. 2015.

[19] L. Xiao, Y. Xiao, Y. Zhao, P. Yang, M. D. Renzo, S. Q. Li and W. Xiang, "Time domain turbo equalization for single-carrier generalized spatial modulation," IEEE Trans. Wireless Commun., vol. 16, no. 9, pp. 5702-5716, Sep. 2017.

[20] P. Liu, K. Luo, D. Chen, and T. Jiang, "Spectral efficiency analysis of cell-free massive MIMO systems with zero-forcing detector," IEEE Trans. Wireless Commun., vol. 19, no. 2, pp. 795-807, Feb. 2020

[21] T. Zhang, C. Wen, S. Jin, and T. Jiang, "Mixed-ADC massive MIMO detectors: Performance analysis and design optimization," IEEE Trans. Wireless Commun. vol. 15, no. 11, pp. 7738-7752, Nov. 2016.

[22] D. Kong, D. Qu, K. Luo, and T. Jiang, "Channel estimation under staggered frame structure for massive MIMO system," IEEE Trans. Wireless Commun., vol. 15, no. 2, pp. 1469-1479, Feb. 2016.

[23] L. Xiao, Y. Xiao, C. Xu, X. Lei, P. Yang, S. Li and L. Hanzo, "Compressive sensing assisted spatial multiplexing aided spatial modulation," IEEE Trans. Wireless Commun., vol. 17, no. 2, pp. 794-807, Feb. 2018.

[24] L. Xiao, P. Xiao, Z. Liu, W. Yu, H. Hass and L. Hanzo, "A compressive sensing assisted massive SM-VBLAST system: Error probability and capacity analysis," IEEE Trans. Wireless Commun., vol. 19, no. 3, pp. 1990-2005, Mar. 2020.

[25] E. Basar, U. Aygolu, E. Panayirci, and H. Poor, "Space-time block coded spatial modulation," IEEE Trans. Commun., vol. 59, no. 3, pp. 823-832, Mar. 2011.

[26] S. Sugiura, S. Chen, and L. Hanzo, "Coherent and differential spacetime shift keying: A dispersion matrix approach," IEEE Trans. Commun., vol. 58, no. 11, pp. 3219-3230, Nov. 2010.

[27] S. Sugiura, S. Chen, and L. Hanzo, "Generalized space-time shift keying designed for flexible diversity-, multiplexing- and complexity-tradeoffs," IEEE Trans. Wireless Commun., vol. 10, no. 4, pp. 1144-1153, Apr. 2011.

[28] C. Xu, T. Bai, J. Zhang, R. G. Maunder, S. Sugiura and Z. Wan and L. Hanzo, "Constant-envelope space-time shift keying," IEEE J. of Sel. Topics in Signal Process., Vo. 13, no. 6, pp. 1387-1402, Oct. 2019.
[29] C. Xu, P. Zhang, R. Rajashekar, N. Ishikawa, S. Sugiura, Z. Wang, and L. Hanzo, "Near-perfect finite-cardinality generalized space-time shift keying," IEEE J. Sel. Areas Commun., Vo. 37, no. 9, pp. 2146-2164, Sept. 2019.

[30] E. Basar, U. Aygolu, E. Panayici and H. V. poor, "Orthogonal frequency division multiplexing with index modulation," IEEE Trans. Signal Process., vol. 61. no. 22, pp: 5536-5549, Nov. 2013.

[31] T. Jiang, M. Guizani, H. Chen, W. Xiang, and Y. Wu, "Derivation of PAPR distribution for OFDM wireless systems based on extreme value theory," IEEE Trans. Wireless Commun., vol.7, no.4, pp. 1298-1305, Apr. 2008

[32] M. Wen, X. Cheng, L. Yang, Y. Li and F. Ji, "Index modulated OFDM for underwater acoustic communications," IEEE Comuun. Mag., vol. 54, no. 5, pp. 132-137, May 2016.

[33] Y. Xiao, S. Wang, L. Dan, X. Lei, P. Yang and W. Xiang, "OFDM with interleaved subcarrier index modulation," IEEE Commun. Lett., vol. 18, no. 8, pp. 1447-1450, Aug. 2014.

[34] E. Basar, "OFDM with index modulation using coordinate interleaving," IEEE Commun. Lett., vol. 4, no. 4, pp. 381-384, Aug. 2015.

[35] M. Wen, Y. Zhang, E. Basar and F. Chen, "Equiprobable subcarrier activation method for OFDM with index modulation," IEEE Commun. Lett., vol. 20, no. 12, pp. 2386-2389, Dec. 2016.

[36] Q. Ma, Y. Xiao, L. Dan, P. Yang, L. Peng and S. Li, "Subcarrier allocation for OFDM with index modulation," IEEE Commun. Lett., vol. 20, no. 12, pp. 2434-2437, July 2016.

[37] R. Fan, Y. J. Yu and Y. L. Guan, "Generalization of orthogonal frequency division multiplexing with index modulation," IEEE Trans. Wireles Commun., vol. 14, no. 10, Oct. 2015.

[38] M. Wen, B. Ye, E. Basar, Q. Li and F. Ji, "Enhanced orthogonal frequency division multiplexing with index modulation," IEEE Trans. Wireless Commun., vol. 16, no. 7, July 2017.

[39] M. Wen, E. Basar, Q. Li, B. Zheng and M. Zhang, "Multiple-mode orthogonal frequency division multiplexing with index modulation," IEEE Trans. Commun., vol. 65, no. 9, pp. 3892-3906, Sep. 2017.

[40] G. Kaddoum, Y. Nijsure and H. Tran, "Generalized code index modulation technique for high-date-rate communication systems," IEEE Trans. Veh. Technol., vol. 65, no. 9, pp. 7000-7009, Sep. 2016.

[41] P. Yang, Y. Xiao, L. Yin, Q. Tang, S. Q. Li, and L. Hanzo, "Hybrid bitto-symbol mapping for spatial modulation," IEEE Trans. Veh. Technol., vol. 65 , no. 7 , pp. 5804-5810, July 2015.

[42] J. Li, M. Wen, X. Cheng, Y. Yan, S. Song and M. H. Lee, "Differential spatial modulation with gray coded antenna activation order," IEEE Commun. Lett., vol. 20, no. 4, pp. 1100-1103, June 2016.

[43] S. Althunibat and R. Mesleh, "A bit-to-symbol mapping scheme for spatial modulation with partial channel state information," IEEE Commun Lett., vol. 21, no. 5, pp. 995-998, May 2017.

[44] M. Tran and S. Kim, "Novel bit mapping for generalized spatial modulation in VLC systems," IEEE Photonics Tech. Lett., vol. 31, no. 15, pp. 1257-1260, Aug. 2019.

[45] S. Saha Ray, Graph theory with algorithms and its applications, ISBN 978-81-322-0750-4.

[46] R. Mesleh and A. Alhassi, "Space modulation techniques," 2018, [Online]. https://onlinelibrary.wiley.com/doi/book/10.1002/9781119375692

[47] G. L. Turin, "The characteristic function of Hermitian quadratic forms in complex normal variables," Biometrika, vol. 47, no. 1/2, pp. 199-201, Jun. 1960. [Online]. Available: http://www.jstor.org/stable/2332977.

[48] Robert T. Short, Computation of Rice and noncentral Chi-squared probabilities, PhaseLocked Systems Technical Report PHS0254, [Online]. Available: http://www.phaselockedsystems.com/NoncentralChiSquared.pdf. 